\title{
Nuclear Astrophysics of Supernovae
}

\author{
J. Cooperstein \\ Physics Department \\ Brookhaven National Laboratory

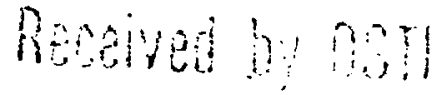 \\ MAY 261989 \\ Lectures \\ Oregon State University \\ Summer School in Nuclear Physics \\ Corvallis, Oregon \\ June 27 - July 8,1988
}

\author{
OUTLINE \\ i: $\quad$ Preface \\ 1: Introduction to Supernova Theory \\ 2: Why does the Star go Unstable? \\ 3: Self-similar Collapse \\ 4: Bounce \\ 5: The Equation of State \\ 6: Neutron Stars \\ 7: $\quad$ Some Final Words
}

This manuscript has been authored under contract number DE-AC02-76CH00016 with the U.S. Department of Energy. Accordingly, the U.S. Government retains a non-exclusive, royaltyfree license to publish or reproduce the published form of this contribution, or allow others to do so, for U.S. Government purposes.

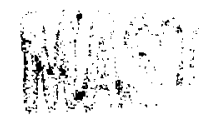




\title{
NUCLEAR ASTROPHYSICS OF SUPERNOVAE
}

\author{
J. Cooperstein \\ Physics Department \\ Brookhaven National Laboratory \\ Upton, New York 11973 \\ U S A \\ i: $\quad$ Preface \\ 1: Introduction to Supernova Theory \\ 2: Why does the Star go Unstable? \\ 3: Self-similar Collapse \\ 4: Bounce \\ 5: The Equation of State \\ 6: Neutron Stars \\ 7: $\quad$ Some Final Words
}

\section{i. PREFACE}

The plan for these lectures is as follows:

First, I'll give a general introduction to Supernova Theory, beginning with the presupernova evolution and ending with the later stages of the explosion. This will be distilled from a colloquium type of talk. It is necessary to have the whole supernova picture in one's mind's eye when diving into some of its nooks and crannies, as it is quite a mess of contradictory ingredients. We will have some discussion of supernova 1987a, but will keep our discussion more general.

Second, we'll look at the infall and bounce of the star, seeing why it goes unstable, what dynamics it follows as it collapses, and how and why it bounces back. From there, we will go on to look at the equation of state (EOS) in more detail. We'll consider the cases $T=0$ and $T>0$. We'll focus on $\rho<\rho_{0}$, and then $\rho>\rho_{0}$ and the EOS of neutron stars, and whether or not they contain cores of strange matter.

There are many things we could discuss here and not enough time. If I had more lectures, the remaining time would focus on two more questions of special interest to nuclear physicists: the electron capture reactions and neutrino transport. If time permited, we'd have some discussion of the nucleosynthetic reactions in the explosion's debris as well. However, we cannot cover such material adequately, and I have chosen these topics because they are analytically tractable, pedagogically useful, and rather important. 
Note added in preparing the written lectures: I have not written up in detail the introductory review lecture with which I began. For a better orientation, the reader could refer to the Hans Bethe's eightieth birthday review volume of Physics Reports 163 (1988). An overview of the problem of neutrinos in supernovae is given in my contribution to that volume. I am also currently writing a review article on the equation of state and the direct mechanism, for the Springer-Verlag volume edited by Albert Petschek. There are other reviews which can be found through the Physics Reports volume and through my references at the end of these notes.

\section{INTRODUCTION}

Many problems in supernova theory pivot upon nuclear astrophysics questions. Strong interactions and weak interactions are involved. We must deal with matter under conditions for the most part inaccessible in the laboratory; i.e., high densities and temperatures, and very neutron rich. The strong force controls the equation of state (EOS). The weak force enters through $\beta$-reactions and through neutrino interactions of various forms. In addition to the problems of extreme conditions, the neutrino physics is complicated because of the non-equilibrium nature of the dynamics. (Time scales are such that the strong and electromagnetic forces are easily equilibrated; the weak interactions are often too slow to achieve chemical or thermal equilibrium.)

I'm in the business of numerical simulations of the supernova process. In this field there has been a constant cycle of analytical and numerical work. The analytical work has taken two distinct forms. The first is microscopic calculations. These are often motivated by modelling requirements. The second is phenomenological analyses of the computer simulations which abstract the crucial quantities and make sense out of the truly awesome quantities of numbers the numerical experiments produce.

In these lectures my emphasis will not be on the microscopic details because of lack of time (as well as expertise) and because my main task is to give you a set of homework problems. There are many ingredients in supernova theory, and often we have felt that the selection of problems we have been able to pursue has been forcibly dictated by gaps in the theoretical framework. Such gaps have taken me personally into subjects such as general relativistic hydrodynamics, generally covariant relativistic kinetic transport theory, strange matter, convection, funny neutrino physics, etc. What I would like to do is to delineate what features are most important and what are most uncertain, and to challenge you to tackle those problems for which you might "have an unfair advantage," (to quote Hans Bethe) so that we simulators can exploit you. 


\section{WHY DOES THE STAR GO UNSTABLE?}

The precise onset of instability is difficult to delineate. Perhaps it is fair to begin during Carbon burning, for at this stage the giant star (be it blue or red) begins emitting more energy in neutrinos than in photons. The neutrinos are mostly $\nu \bar{\nu}$ pairs produced by plasmon processes. Uniike the photons, the neutrinos leave directly providing a central energy sink. The star's subsequent burning stages are correspondingly quite short, the final stage of silicon burning to iron peak elements taking only hours or days.

Simulations of the supernova explosion are generally broken into two stages. The presupernova massive star evolution codes are terminated when the central density reaches $\sim 10^{9} \mathrm{gm} / \mathrm{cm}^{3}$. At this point the middle of the iron core is already collapsing at $v \sim-10^{8} \mathrm{~cm} / \mathrm{sec}$. The following stages take only seconds. The presupernova codes cannot follow the subszquent evolution because they do not include general relativity, neutrino transport, or denser matter equations of state. These begin to be sizeable corrections, and new codes must be used and new physics must he incorporated. Thus begins the second stage of the supernova process which groups like ours then follow, the explosion itself.

What are conditions like at this point? At the center we have:

$$
\rho \sim 10^{10} \mathrm{gm} / \mathrm{cm}^{3} ; T \leq 1 \mathrm{MeV} ; Y_{e} \sim 0.42
$$

where $Y_{e}$ is the number of protons per baryon. By charge conservation we have

$$
Y_{e}=Y_{e^{-}}-Y_{e^{+}}
$$

for the net lepton number, there being no appreciable number of neutrinos as yet. Under such conditions the pressure is dominated by relativistic electrons, and thus the center of the star looks like a (hot) white dwarf. To see this we must look first at the electron-positron gas equation of state. The net density of relativistic electron-positron pairs $\left(m_{e}=0\right)$ is given by

$$
n_{e}=\rho Y_{e}=2 \int \frac{d^{3} k}{(2 \pi)^{3}}\left(f_{e^{-}}-f_{e^{+}}\right)
$$

where $f_{e^{ \pm}} \equiv f\left(\frac{k \mp \mu_{e}}{T}\right)=\left(1+\exp \left[\frac{k \mp \mu_{e}}{T}\right]\right)^{-1}$, the 2 being for spin, and $\mu_{e}=$ $\mu_{e^{-}}=-\mu_{e^{+}}$is the electron chemical potential. (We will take $\hbar=c=1$ ) The distributions are isotropic, and thus

where

$$
n_{e}=\frac{T^{3}}{\pi^{2}}\left(F_{2}\left(\eta_{e}\right)-F_{2}\left(-\eta_{e}\right)\right)
$$




$$
\eta_{e}=\mu_{e} / T
$$

is the electron degeneracy parameter, and the conventional Fermi integral is defined as

$$
F_{n}(\eta) \equiv \int_{0}^{\infty} d x x^{n} f(x-\eta) .
$$

Similarly the energy density of the electron positron gas is given by

$$
\begin{aligned}
& \varepsilon_{e}=\varepsilon_{e^{-}}+\varepsilon_{e^{+}}=2 \int \frac{d^{3} k}{(2 \pi)^{3}} k\left(f_{e^{-}}+f_{e^{+}}\right) \\
& \varepsilon_{e}=\frac{T^{4}}{\pi^{2}}\left(F_{3}\left(\eta_{e}\right)+F_{3}\left(-\eta_{e}\right)\right)
\end{aligned}
$$

Irrespective of the state of degeneracy, we have the relativistic relation

$$
P_{e}=\frac{1}{3} \varepsilon_{e} .
$$

We see we have to do integrals of the form

$$
G_{n}(\eta) \equiv F_{n}(\eta)-(-1)^{n} F_{n}(-\eta)
$$

Now $G_{0}$ is elementary:

$$
\begin{aligned}
G_{0}(\eta) & =\int_{0}^{\infty} \frac{d x}{1+e^{x-\eta}}-\int_{0}^{\infty} \frac{d x}{1+e^{x+\eta}} \\
& =\ln \left(1+e^{\eta}\right)-\ln \left(1+e^{-\eta}\right)=\eta
\end{aligned}
$$

exactly. Noting that

$$
\begin{aligned}
\frac{d}{\partial \eta} F_{n}(\eta) & =\int_{0}^{\infty} d x x^{n} \frac{f(x-\eta)}{\partial \eta}=-\int_{0}^{\infty} d x x^{n} \frac{\partial}{\partial x} f(x-\eta) \\
& =-x^{n} f(x-\eta)+n \int_{0}^{\infty} d x x^{n-1} f(x-\eta)=n F_{n-1}(\eta)
\end{aligned}
$$

for $n>0$, or equivalently (being careful with signs!)

$$
\frac{\partial G_{n}(\eta)}{\partial \eta}=n G_{n-1}(\eta)
$$

we can build up the $G$-functions by recursive integration; i.e.,

$$
G_{1}(\eta)=\int d \eta G_{0}(\eta)=\frac{\eta^{2}}{2}+C_{1}
$$


The integration constant is obtained by

$$
\begin{aligned}
C_{1} & =2 F_{1}(0)=2 \int_{0}^{\infty} \frac{d x x}{1+e^{x}}=2 \int_{0}^{\infty} d x x e^{-x}\left(1-e^{-x}+e^{-2 x}+\cdots\right) \\
& =2\left(1-\frac{1}{2^{2}}+\frac{1}{3^{2}}-\frac{1}{4^{2}}+\cdots\right)=2 \cdot \frac{\pi^{2}}{12}=\frac{\pi^{2}}{6}
\end{aligned}
$$

and we can continue. (Note $C_{0}=C_{2}=C_{4}=\cdots=0$.) The first few $G$-functions are:

$$
\begin{aligned}
& G_{0}=\eta \\
& G_{1}=\frac{\eta^{2}}{2}+\frac{\pi^{2}}{6} \\
& G_{2}=\frac{\eta^{3}}{3}+\frac{\pi^{2} \eta}{3} \\
& G_{3}=\frac{\eta^{4}}{4}+\frac{\pi^{2} \eta^{2}}{2}+\frac{7 \pi^{4}}{60} .
\end{aligned}
$$

It is possible to write an expression for $G_{n}$ in closed form, but it is a hideous mess of Bernoulli numbers or Euler's zeta function. (This digression may seem extended, but Fermi integral technology is an essential implement in the astrophysical toolbox.) Thus we have obtained

$$
\begin{aligned}
& \rho Y_{e}=\frac{\mu_{e}^{3}}{3 \pi^{2}}\left(1+x^{2}\right) \\
& \varepsilon_{e}=3 \rho_{e}=\frac{\mu_{e}^{4}}{4 \pi^{2}}\left(1+2 x^{2}+\frac{7}{15} x^{4}\right)
\end{aligned}
$$

where $x \equiv \pi / \eta_{e}$, which shows that degeneracy should be measured by $\eta_{e} \gg \pi$. Note that the average energy of an electron is given by

$$
\frac{\varepsilon_{e}}{\rho Y_{e}}=\frac{3}{4} \mu_{e}\left(\frac{1+2 x^{2}+\frac{7}{15} x^{4}}{1+x^{2}}\right)
$$

(This doesn't make sense when there are a lot of positrons around, $\mu_{e} \sim 0$.)

Focusing on warm, but not completely degenerate matter, we see that the chemical potential, $\mu_{e}$ is related to the electron Fermi energy, $\varepsilon_{F}$, by

$$
\begin{aligned}
& \varepsilon_{F}^{3}=\mu_{e}^{3}\left(1+x^{2}\right) \\
& \varepsilon_{F}=\left(3 \pi^{2} \rho Y_{e}\right)^{1 / 3} \hbar c .
\end{aligned}
$$


Letting $\rho=10^{10} \rho_{10} \mathrm{gm} / \mathrm{cm}^{3}=6.02 \cdot 10^{-6} \rho_{10} \mathrm{fm}^{-3}$,

$$
\begin{aligned}
\varepsilon_{F} & =\left(3 \pi^{2} \cdot 6.02 \cdot 10^{-6} \cdot 0.42\right)^{1 / 3} 197.33\left(\frac{Y_{e}}{0.42} \rho_{10}\right)^{1 / 3} \mathrm{MeV} \\
& =8.32\left(\rho_{10} \frac{Y_{e}}{0.42}\right)^{1 / 3} \mathrm{MeV} .
\end{aligned}
$$

Since initially $T \lesssim 1 \mathrm{MeV}$, we see that $\eta_{e} \gg \pi$ and the thermal corrections are smali to the electron EOS. Note that the entropy per nucleon in electrons, $S_{e}$, can be obtained from the basic thermodynamic relation,

$$
T S_{e}+Y_{e} \mu_{e}=\left(\varepsilon_{e}+P_{e}\right) / \rho,
$$

so

$$
\begin{aligned}
S_{e} & =\frac{4}{3} \frac{\varepsilon_{e}}{\rho T}-Y_{e} \eta_{e}=Y_{e}\left[\frac{4}{3} \frac{\varepsilon_{e}}{\rho Y_{e} T}-\eta_{e}\right] \\
& =Y_{e} \eta_{e}\left(\frac{1+2 x^{2}+(7 / 15) x^{4}}{1+x^{2}}-1\right)=Y_{e} \eta_{e}\left(\frac{\pi}{\eta_{e}}\right)^{2}\left(1+\frac{7}{15} x^{2}\right)
\end{aligned}
$$

So in the limit that $x \gg 1\left(\eta_{e} \gg \pi\right)$

$$
S_{e} \simeq Y_{e} \frac{\pi^{2}}{\eta_{e}}=0.50 \rho_{10}^{-1 / 3}\left(\frac{Y_{e}}{0.42}\right)^{2 / 3} T .
$$

Note that

$$
x=\frac{\pi}{\eta_{e}}=\frac{S_{e}}{\pi Y_{e}} .
$$

Assuming the thermal corrections to be small, we find

$$
\mu_{e}=\varepsilon_{F}\left(1-1 / 3 x^{2}\right) .
$$

It is instructive to consider the pressure per nucleon in electrons,

$$
\frac{P_{e}}{\rho}=Y_{e} \frac{P_{e}}{\rho Y_{e}} \cong \frac{Y_{e} \mu_{e}}{4}\left(\frac{1+2 x^{2}}{1+x^{2}}\right) \cong \frac{Y_{e} \varepsilon_{F}}{4}\left(1+\frac{2}{3}\left(\frac{S_{e}}{\pi Y_{e}}\right)^{2}\right) \text {. }
$$

Putting in numbers

$$
\frac{P_{e}}{\rho}=0.87\left(\frac{Y_{e}}{0.42}\right)^{4 / 3} \rho_{10}^{1 / 3}\left(1+\frac{2}{3}\left(\frac{S_{e}}{\pi Y_{e}}\right)^{2}\right) \mathrm{MeV} .
$$


If the electrons were a non-degenerate Maxwell-Boltzmann gas their pressure would be

so

$$
\frac{P_{e}}{\rho}=Y_{e} T \quad(\mathrm{MB})
$$

$$
\frac{P_{e}}{P_{e}(\mathrm{MB})}=\frac{\varepsilon_{F}}{4 T}=\frac{n_{e}}{4} .
$$

Now the pressure due to nucleons is given by

$$
\frac{P_{N}}{\rho}=\sum_{A, Z} \frac{\phi_{A, Z}}{A} T+\frac{P_{\text {coul }}}{\rho}=Y T+\frac{P_{\text {coul }}}{\rho},
$$

where $\phi_{A, Z}$ is the mass fraction of the $(A, Z)$ nucleus normalized so that

$$
1=\sum \phi_{A, Z}
$$

$\mathrm{Y}$ is the number of independent particles per nucleon, and $P_{\text {coul }}$ is the coulomb lattice pressure and is negative. (We will discuss this later in lecture 5.) It is given roughly by

$$
\frac{P_{\text {coul }}}{\rho} \cong-0.036 \rho_{10}^{1 / 3}
$$

(for $Y_{e} \sim 0.42$ and nucleons clustered mostly in one large nuclear species) Thus,

$$
\frac{P_{\text {coul }}}{P_{e}} \simeq-\frac{0.036}{0.86} \simeq-0.04
$$

(Note $P_{\text {coul }}$ and $P_{e}$ have the same density dependence, both varying inversely with distance)

The nuclear thermal pressure, $Y T$, depends on $Y$. For example, consider a mixture of $10 \%$ free nucleons and iron. Then

$$
Y=0.1 \cdot\left(\frac{1}{1}\right)+0.9 \cdot\left(\frac{1}{56}\right)=0.116
$$

and for pure iron. $Y=1 / 56=0.018$. The two cases would give

$$
\frac{Y T}{P_{e} / \rho}=\frac{Y T}{Y_{e} \mu_{e} / 4} \cong \frac{Y T}{0.87} \sim(0.02-0.13) T
$$

the $2 \%$ correction being more appropriate. Thus, the nuclear contribution to the pressure is smaller than the electron one, and can even be negative if all the nucleons are in one large nucleus. Thus, the electron pressure dominates.

In Figure 1, we plot $Y$ and $P_{N} / P_{e}$, and see they are indeed quite small. Thus, to first approximation the equation of state is dominated by the electrons. 

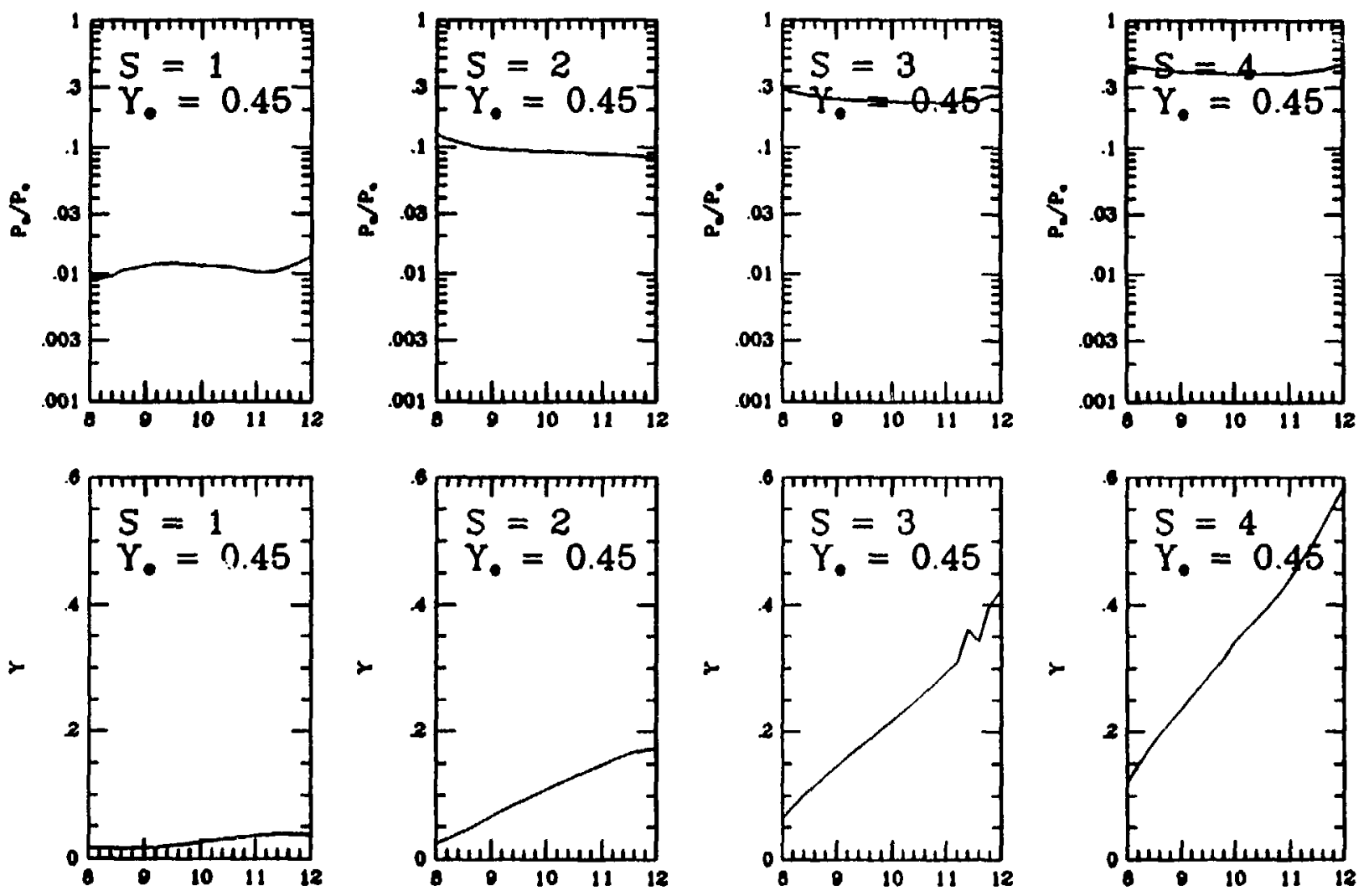

Fig. 1. Relative pressures and composition of infalling supernova matter. The top curves gives $P_{N} / P_{e}$, the ratio of nucleonic to leptonic pressure, and the bottom curves give $Y$, the number of independent particles per nucleon, along the adiabats $S=1,2,3,4$, as a funcion of density. All curves are for the case $Y_{e}=0.45$, as are figures $2,4,5$. 

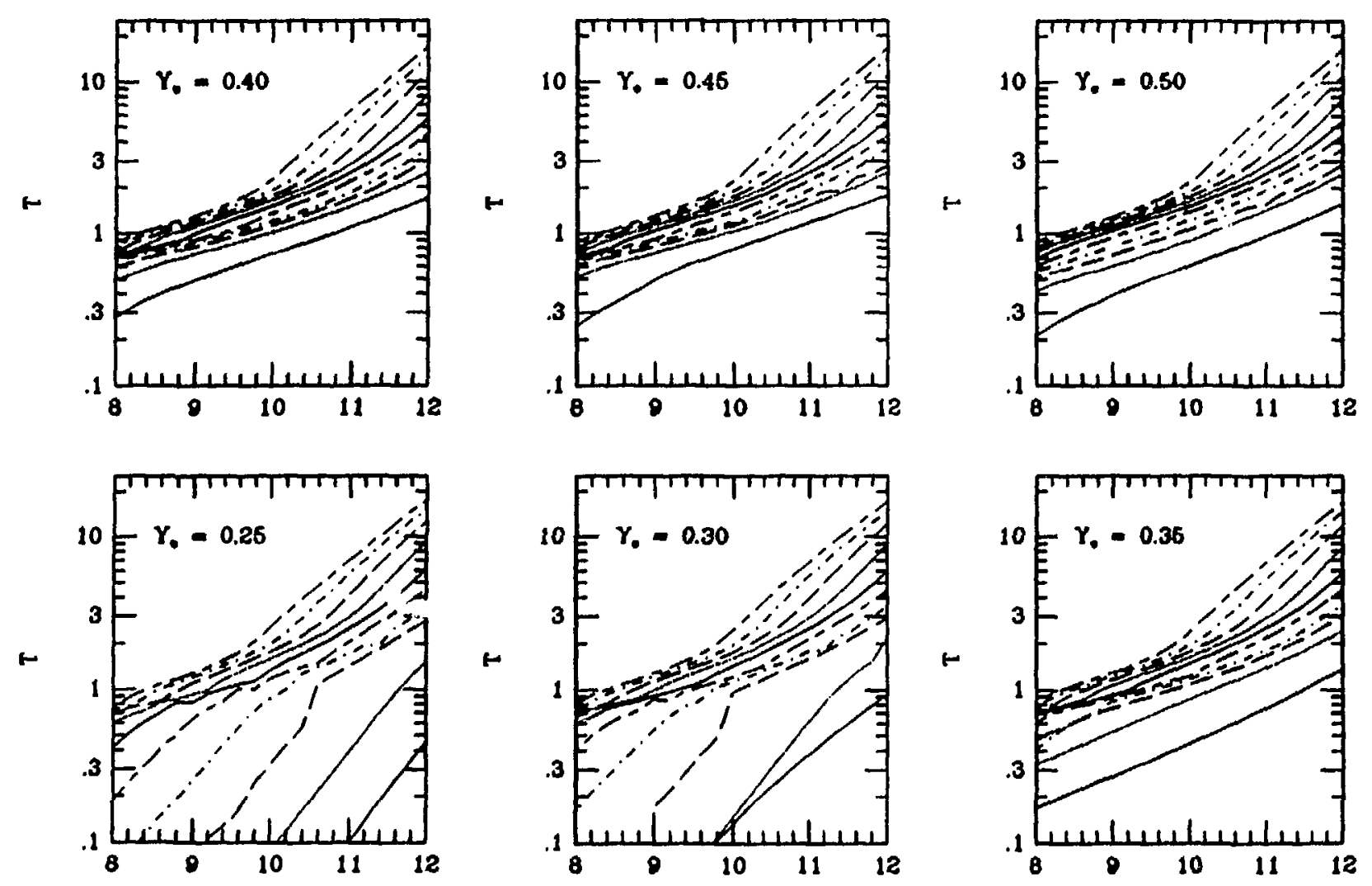

Fig. 2. Temperature of supernova matter. The temperature is given in $\mathrm{MeV}$ for adiabats $S=$ $1,2 \ldots 10 . S=1,6$ is denoted by a solid line; $S=3,8$ by a long dashed line, etc. The cases $Y_{e}=0.25,0.30, \ldots, 0.5$ are given. 

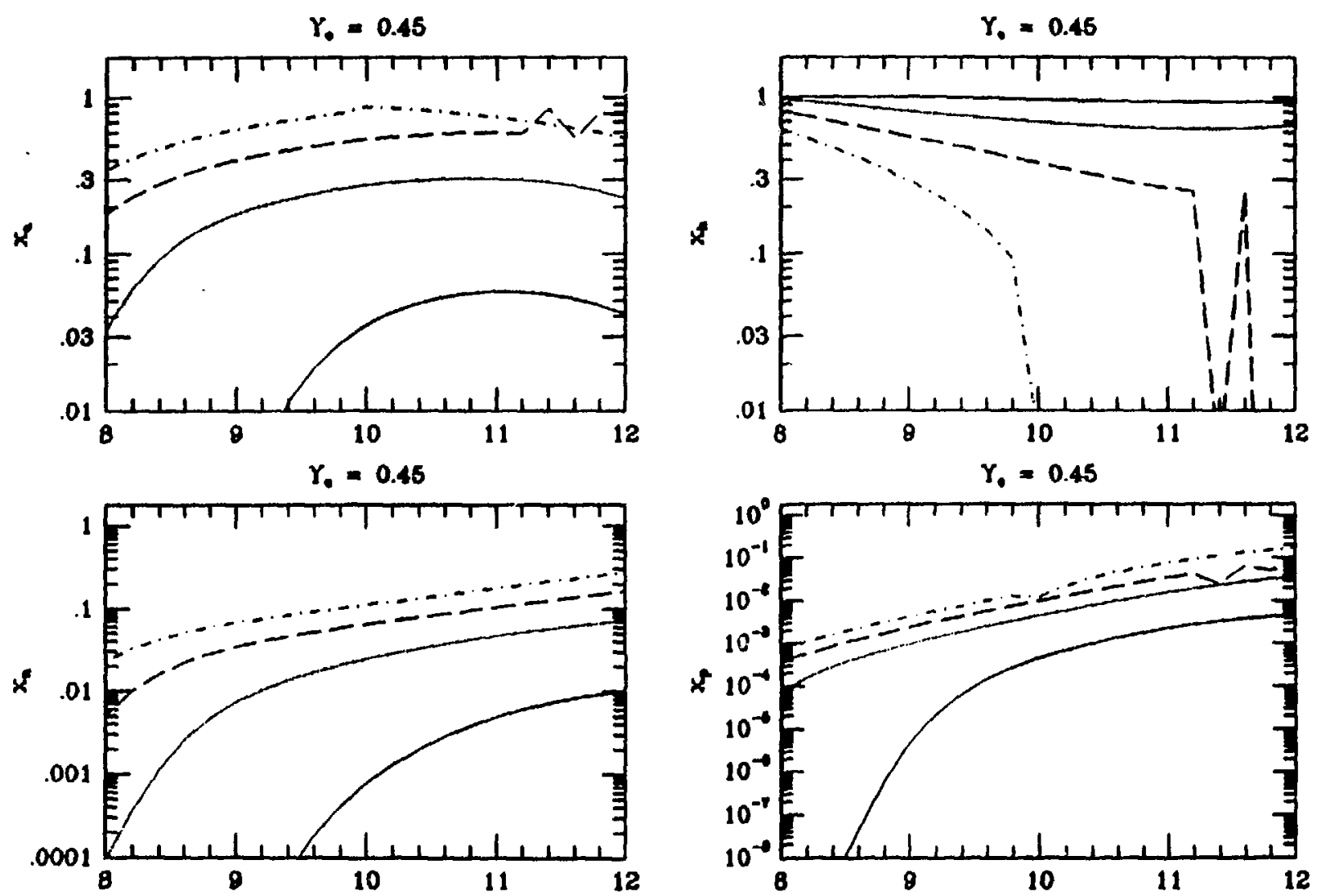

Fig. 3. Composition of supernova matter. Particle abundances by mass function for $Y_{e}=0.45$, early in supernova collapse. Shown are $X_{a}, X_{h}, X_{n}$, and $X_{p}$, the mass fractions of alpha particles, heavy nuclei, and free neutrons and protons, for $S=1,2, \ldots, 10 . S=1,6$ is denoted by a solid line; $S=3,8$ by a long dashed line, etc. 
Figure 2 shows the temperature as a function of $\rho, Y_{c}$, and $S$; figure 3 shows the mass fractions $X_{n}, X_{p}, X_{\alpha}$, and $X_{H}$; and figure 4 shows the adiabatic index $\Gamma$. There's a lot of information in these figures, but they are noisy and so don't take all the wiggles seriousiy, as I was too lazy to generate a smooth table.

Now finally we can begin to ask why the star goes unstable. With Newtonian gravity a relativistic star has zero total energy. This is because both the internal energy and the gravitational energy vary inversely with distance and thereby cancel. This is a standard result of polytrope theory. This means the entire star can be shrunk or expanded with no cost in energy, accordion style. Thus, small perturbations can lead to runaway instabilities. In the case of a white dwarf star, the outermost regions are not so relativistic, and they provide the stability.

Two processes work hand in hand to propel collapse. These are electron capture and photodisintegration. We will consider each.
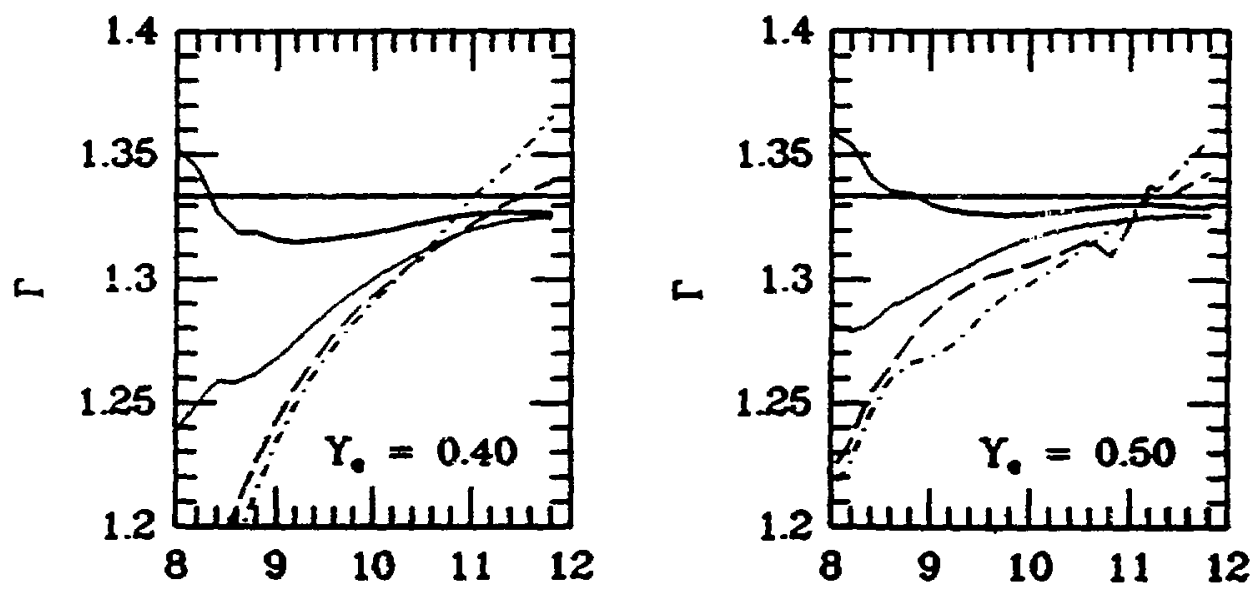

Fig. 4. Adiabatic index. $\Gamma=d \ln P /\left.d \ln \rho\right|_{0}$ is displayed for $Y_{e}=0.40$ and 0.50 for $S=1$ (solid line), $S=2$ (dotted line), $S=3$ (dashed line), and $S=4$ (dashed-dotted line). The value $\gamma=4 / 3$ is also shown. Note that the $S=1$ line drops below $4 / 3$ at $\rho \sim 10^{8.5} \mathrm{gm} / \mathrm{cm}^{3}$, due to photodisintegration.

In any regime one may approximate the EOS by the polytropic power law

$$
p=K p^{\gamma}
$$

where the pressure constant, $K$, and the adiabatic exponent, $\gamma$, are considered independent of space and time. For the relativistic gas $\gamma=4 / 3$. Now there exist various kinds of $\gamma^{\prime} s$ that enter into supernova collapse, the most important one being

$$
\left.\Gamma_{\mathrm{ad}} \equiv \frac{d \ln P}{d \ln \rho}\right|_{S, Y_{e}},
$$


the derivative taken with $S$ and $Y_{e}$ held constant. (If neutrinos are present, and in equilibrium with the matter, $Y_{e}+Y_{\nu_{e}}$ should be held constant.) This $\Gamma_{a d}$ is purely a function of the equation of state. However, another critical adiabatic index is provided by

$$
\Gamma_{\text {coll }}=\left.\frac{d \ln P}{d \ln \rho}\right|_{M}
$$

i.e., the derivative is taken as a mass element collapses. The relationship between the two can be written as

$$
\Gamma_{\mathrm{coll}}=\Gamma_{\mathrm{ad}}+\left.\frac{\partial \ln P}{\partial S}\right|_{Y_{e}} \frac{\delta S}{\delta \ln \rho}+\left.\frac{\partial \ln P}{\partial \ln Y_{e}}\right|_{S} \frac{\delta \ln Y_{e}}{\delta \ln \rho},
$$

giving the value of $\Gamma_{\text {coll }}$ over a small change $\delta \ln \rho$. If we try to approximate the EOS with the polytropic law and consider $\gamma \sim \Gamma_{\text {coll }}$ and for the moment ignore the change in entropy due to the process, we would find (ignoring the nucleons)

$$
\gamma \sim \Gamma_{\text {coll }} \sim \frac{4}{3}\left(1+\frac{\delta \ln Y_{e}}{\delta \ln \rho}\right) .
$$

Now, we observe that in the supernova the process

$$
e^{-}+p \rightarrow n+\nu_{e}
$$

drops $Y_{e}$ from 0.45 to 0.40 as $\rho_{10}$ increases from 1 to 100 , and thus we find

$$
\gamma \sim \frac{4}{3}\left(1+\frac{\ln (0.40 / 0.45)}{\ln (100)}\right) \simeq \frac{4}{3}(1-0.0256)=1.30 .
$$

Actually it is $K$ which has dropped, but we can approximate this by a decrease in $\gamma$. So electron capture decreases $\gamma$ to less than $4 / 3$.

Photodisintegration involves reactions of the type

$$
(A, Z)+\gamma \rightarrow\left(\frac{Z}{2}\right) \alpha+(A-2 Z) n
$$

or in the case of iron

$$
\mathrm{Fe}^{56} \rightarrow 13 \alpha+4 n,
$$

which is endothermic, costing $\sim 124 \mathrm{MeV}$. But why do these reactions take place as the star collapses? Stellar evolution involves a series of burning stages of successive fuels: $\mathrm{H}, \mathrm{He}, \mathrm{C}, \mathrm{O}, \mathrm{Ne}, \mathrm{Si}$, etc. After each stage exhausts its fuel, the center contracts and heats up, and the ashes of the previous burning stage become the fuel for the next one. This process terminates at iron (actually in the "iron peak") where no more energy can be gained by fusion. Subsequently, the star contracts looking for more fuel, but not finding any actually induces fission through gamma 

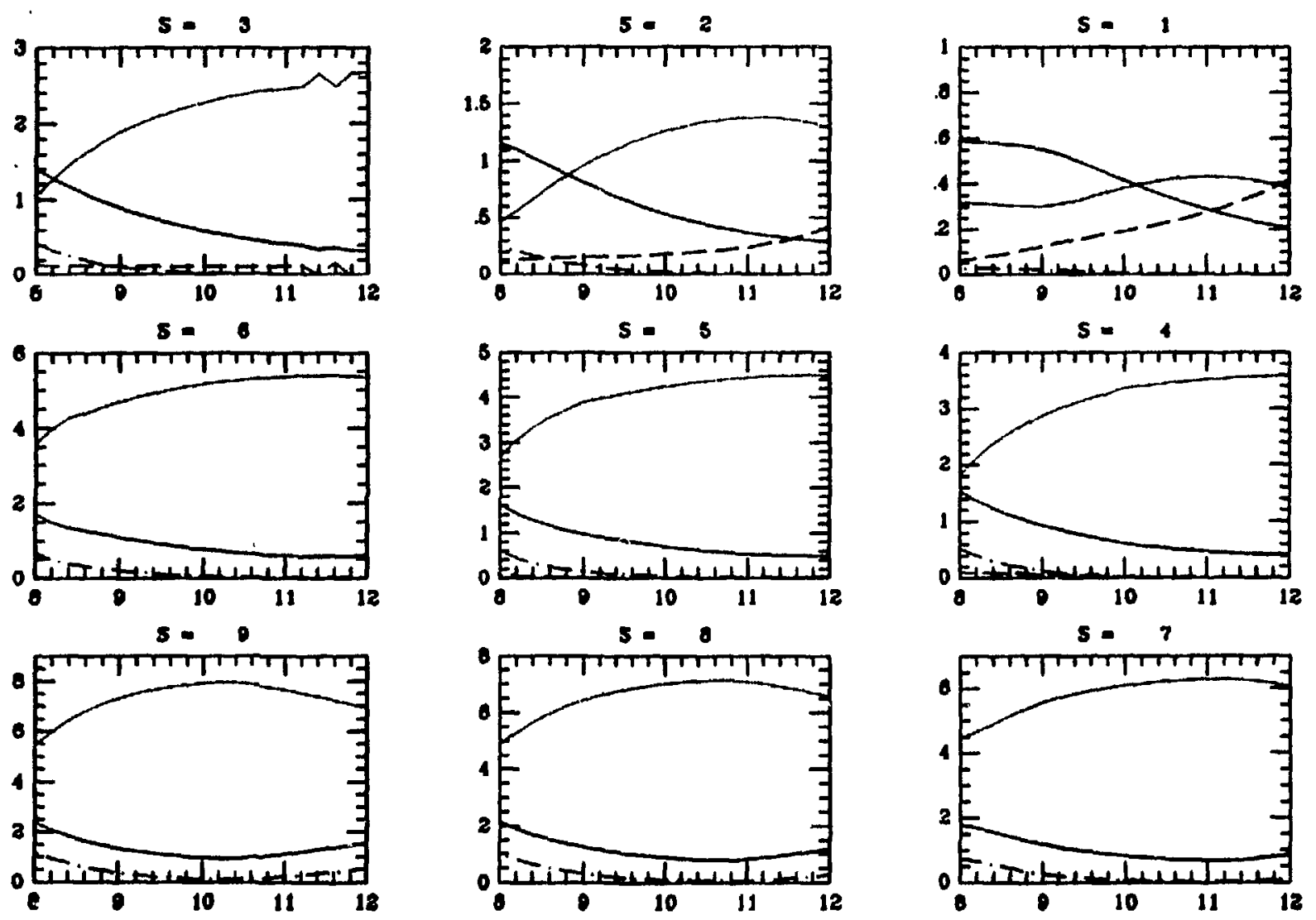

Fig. 5. Contributions to the entropy. Displayed are $S_{e}$, the electron entropy (solid line), $S_{0}$ the entropy in baryonic translational motion (dotted line), $S^{*}$, the entropy stored in nuclear excited states (dashed line), and $S_{\mathrm{RAD}}$, the entropy in photons (dashed-dotted line), for the adiabats $S=$ $1,2, \ldots, 10$, for the case $Y_{c}=0.45$. Note that as the density increases along the adiabats, entropy is transfered from the electrons to the nucleons. 
rays as the temperature increases with an insufficient concommitant rise in the pressure. This energy (the $124 \mathrm{MeV}$ for iron) must come from sonewhere, and where it comes from is the electrons. Remember that

$$
\frac{P_{c}}{\rho}=\frac{Y_{e} \varepsilon_{F}}{4}\left(1+\frac{2}{3}\left(\frac{S_{c}}{\pi Y_{e}}\right)^{2}\right)
$$

so that for the pure electron gas

$$
\Gamma_{\mathrm{ad}}=\left.\frac{\partial \ln P_{e}}{d \ln \rho}\right|_{\mathcal{S}_{E}}=\frac{4}{3}
$$

irrespective of the degeneracy, or $S_{e}$. But the photodisintegration reaction reduces $S_{e}$ by transferring entropy from the electrons to nucleons, because an iron nucleus disintegrated into constituents carries much more entropy per nucleon than if it stays in one piece.

Now,

$$
\begin{aligned}
\Gamma_{\mathrm{ad}}=\left.\frac{\partial \ln P}{\partial \ln \rho}\right|_{S} & =\frac{P_{e}}{P_{e}+P_{N}}\left[\left.\frac{\partial \ln P_{e}}{\partial \ln \rho}\right|_{S_{e}}+\left.\frac{\partial \ln P_{e}}{\partial S_{e}} \frac{\delta S_{e}}{\delta \ln \rho}\right|_{S}\right] \\
& +\frac{P_{N}}{P_{e}+P_{N}}\left[\left.\frac{\partial \ln P_{N}}{\partial \ln \rho}\right|_{S_{N}}+\left.\frac{\partial \ln P_{N}}{\partial S_{N}} \frac{\delta S_{N}}{\partial \ln \rho}\right|_{S}\right]
\end{aligned}
$$

at fixed $Y_{e}$. Ignoring the second term, the one due to nucleons, we find

$$
\Gamma_{\mathrm{ad}} \simeq \frac{1}{1+\frac{P_{N}}{P_{e}}} \frac{4}{3}\left[1+\frac{\left(\frac{S_{e}}{\pi Y_{e}}\right)^{2}}{1+\frac{2}{3}\left(\frac{S_{e}}{\pi Y_{e}}\right)^{2}} \frac{1}{S_{e}} \frac{\delta S_{e}}{\delta \ln \rho}\right] .
$$

Looking at figure 5 we see that the second term is indeed negative along adiabats, and thus the effective $\Gamma$ is reduced. Thus, photodisintegration, which actually cools the electrons as matter heats up, promotes instability.

How does the collapse proceed?

\section{SELF-SIMILAR HOMOLOGOUS COLLAPSE}

Here we will follow the beautiful work of Amos Yahil (Ap. J. 265 (1983) 1047) who considered the case $1.2 \leq \gamma \leq 4 / 3$, and was motivated by the earlier and also quite elegant work of Goldreich and Weber (Ap. J. 238 (1980) 991).

Yahil notes there is only a small charge in the entropy during the collapse, and suggests the polytropic equation of state should suffice at describing at least the early stages of the problem. He also notes that, thus, there are only two 
dimensional constants in the problem, $K$ and $G$, and that only one dimensionless combination of $r$ and $t$ can be made with them, which is linear in $r$ :

$$
X=K^{-1 / 2} G^{(\gamma-1) / 2} r(-t)^{\gamma-2},
$$

the minus sign occurring with $t$ because $t=0$ is taken to be the moment of catastrophe, or infinite central density. The idea is that any other parameters in the problem besides $K$ and $G$, such as the initial density and velocity, will have only a transient effect, and thus all the hydrodynamical variables (density, velocity, energy, etc) should only be functions of the self-similar variable $X$. Yahil says, "Thus, the solutions are self-similar; i.e., they h.dve the same spatial structure at all times." Goldreich and Weber did consider, in the more limited case of $\gamma=4 / 3$, whether perturbations to the ordered flow would amplify faster than conditions changed due to collapse, and found their solutions to be stable. In the more general case Yahil's conjecture is well borne out by many numerical simulations.

Yahil contirues to construct the dimensionless hydrodynamical variables:

$$
\begin{aligned}
& \rho=G^{-1}(-t)^{-2} D(X) \\
& v=K^{1 / 2} G^{(1-\gamma) / 2}(-t)^{1-\gamma} V(X) \\
& m=K^{3 / 2} G^{(1-3 \gamma) / 2}(-t)^{4-3 \gamma} M(X) \\
& \varepsilon=K^{5 / 2} G^{(3-5 \gamma) / 2}(-t)^{6-5 \gamma} E(X)
\end{aligned}
$$

where $\rho, v, m, \varepsilon(D, V, M, E)$ are the dimensional (dimensionless) density, velocity, enclosed mass, and enclosed energy. These last two are defined by

$$
\begin{aligned}
& M(X)=\int_{0}^{X} d X 4 \pi X^{2} D(X) \\
& E(X)=\int_{0}^{X} d X 4 \pi X^{2} D(X)\left[\frac{V^{2}(X)}{2}+\frac{D(X)^{\gamma-1}}{(\gamma-1)}-\frac{M(X)}{X}\right] .
\end{aligned}
$$

He then must put the acceleration equation

$$
\frac{d v}{d t}+\frac{1}{\rho} \frac{\partial P}{\partial r}+\frac{G m}{r^{2}}=0
$$

and the equation of continuity

$$
\frac{\partial \rho}{\partial t}+\frac{1}{r^{2}} \frac{\partial}{\partial r}\left(r^{2} \rho v\right)=0
$$

into dimensionless form. (In doing this it is important to use the proper convective derivative; i.e., $\frac{d v}{d t}=\frac{\partial v}{\partial t}+v \frac{\partial v}{\partial r}$.) He finds the two coupled equations

$$
\begin{aligned}
& {[V-(\gamma-2) X] D^{\prime} / D+V^{\prime}=-(2+2 V / X)} \\
& \gamma D^{\gamma-2} D^{\prime}+[V-(\gamma-2) X] V^{\prime}=-\left(M / X^{2}+(\gamma-1) V\right)
\end{aligned}
$$


where a prime indicates differentiation with respect to $X$. He next breaks the dimensionless velocity into two pieces

$$
V=(\gamma-2) X+U
$$

This is an important step. If $V=(\gamma-2) X$ then a fluid element remains stationary in that frame; i.e., always has the same $x$ coordinate. (See the defining relation for $X \sim r(-t)^{\gamma^{-2}}$.) Thus, the "wind velocity" $U$ is measured with respect to a co-moving, homologous frame. Substituting $U$ into the equations gives

$$
\begin{aligned}
& \frac{U}{D} D^{\prime}+U^{\prime}=(4-3 \gamma)-\frac{2 U}{X} \\
& \gamma D^{\gamma-2} D^{\prime}+U U^{\prime}=-\frac{M}{X^{2}}+(\gamma-1)(2-\gamma) X+(3-2 \gamma) U .
\end{aligned}
$$

The first equation can be easily integrated by multiplying by $4 \pi X^{2} D$ and integrating from 0 to $X$, resulting in

$$
4 \pi X^{2} D U=(4-3 \gamma) M .
$$

Note that in the case $\gamma=4 / 3, U$ vanishes everywhere. This means the entire core collapses homologously. But for $\gamma\langle 4 / 3, U>0$; the interpretation is that the outer core is unable to keep up with the inner core. It is this feature incorporated into the Yahil theory that represents a great improvement over the earlier Goldreich-Weber theory. In the latter case, a limiting homologous core mass, equal to $1.45 \mathrm{M}_{\mathrm{ch}}$, was found corresponding to a boundary condition of the outer edge being in free fall. Yahil's theory extends to larger core masses as we indeed find in supernova theory. (The initial iron core Chandresekhar mass is $\mathrm{M}_{\mathrm{ch}} \sim 1.45 M_{\odot}$, but due to electron capture it drops to $\sim 0.9 M_{\odot}$, and thus there truly is an inner and outer core).

It is important to get the asymptotic behavior of the hydrodynamical variables as $X \rightarrow \infty(r \rightarrow \infty$ or $-t \rightarrow 0)$. In order to avoid singularities in this case, we must note here that the $t$-dependence of these variables must be cancelled by the $t$-dependence implicit in the $X$-dependence of the dimensionless variables. This results for $X \gg 1(r \gg 0)$ in

$$
\begin{array}{ll}
D(X) \sim X^{-2 /(2-\gamma)} & \rho(r) \sim r^{-2 /(2-\gamma)} \\
M(X) \sim X^{(4-3 \gamma) /(2-\gamma)} & m(r) \sim r^{(4-3 \gamma) /(2-\gamma)} \\
V(X) \sim X^{(1-\gamma) /(2-\gamma)} & v(r) \sim r^{(1-\gamma) /(2-\gamma)} \\
E(X) \sim X^{(6-5 \gamma) /(2-\gamma)} & \varepsilon(r) \sim r^{(6-5 \gamma) /(2-\gamma)} .
\end{array}
$$

At the time of catastrophe this gives the asymptotic behavior of the entire infalling matter. However, by this point the self-similar solution breaks down at 
the center because the stiffening of the equation of state above nuclear density introduces new dimensional parameters into the theory. (Note $\gamma \leq 1.2$ is excluded because the energy diverges in this case.)

Let's consider the asymptotic behavior of several important quantities at large $r$. For $\gamma=4 / 3$ and $\gamma=1.3$ we find:

Table 1 - Asymptotic Behavior

\begin{tabular}{|c|c|c|}
\hline & $\gamma=4 / 3$ & $\gamma=1.3$ \\
\hline$\rho$ & $r^{-1 / 3}$ & $r^{-0.28}$ \\
$v_{f f}=2 G m / r$ & $r^{1 / 2}$ & $r^{0.42}$ \\
$v / v_{f f}$ & $r^{-1}$ & $r^{-0.86}$ \\
$\rho v^{2}$ & $r^{3 / 2}$ & $r^{1.29}$ \\
\hline
\end{tabular}

We see that as $\gamma$ is reduced, the density in the outer core drops less slowly with radius, because the inner core falls away more rapidly. We shall see that this will be harmful to the shock.

To proceed furtter, we really need to get the constants in front of the asymptotic relationships. This means we must integrate the differential equation. Since we have eliminated $V$, we must integrate

$$
\begin{aligned}
& \frac{d M}{D X}=4 \pi X^{2} D \\
& \frac{d D}{D X}=D\left[(1-\gamma) U-\frac{2 U^{2}}{X}+\frac{M}{X^{2}}-(\gamma-1)(2-\gamma) X\right] /\left[U^{2}-\gamma D^{\gamma-1}\right] .
\end{aligned}
$$

Now at small $X \ll 1$,

$$
U=(4 / 3-\gamma) X, \quad M=\frac{4 \pi}{3} X^{3} D_{0},
$$

where $D_{0}$ is the dimensionless central density. Note that the dimensionless sound speed is given by

$$
A=\gamma^{1 / 2} D^{(\gamma-1) / 2},
$$

and thus at small $X$ the wind velocity is subsonic. At large $X$ we have the asymptotic relation,

$$
A \sim X^{(1-\gamma) /(2-\gamma)} ; X \gg 1 .
$$

But since for $X \gg 1, U \rightarrow \infty$ because $V \rightarrow 0$, the wind becomes supersonic. Note that in the differential equation for $D$, the denominator vanishes at the critical point, or sonic point, where the wind becomes supersonic. Yahil finds the correct solution to the differential equation by varying $D_{0}$. If $D_{0}$ is too low, the wind velocity $U$ reaches a subsonic maximum and then falls to zero at infinity, 

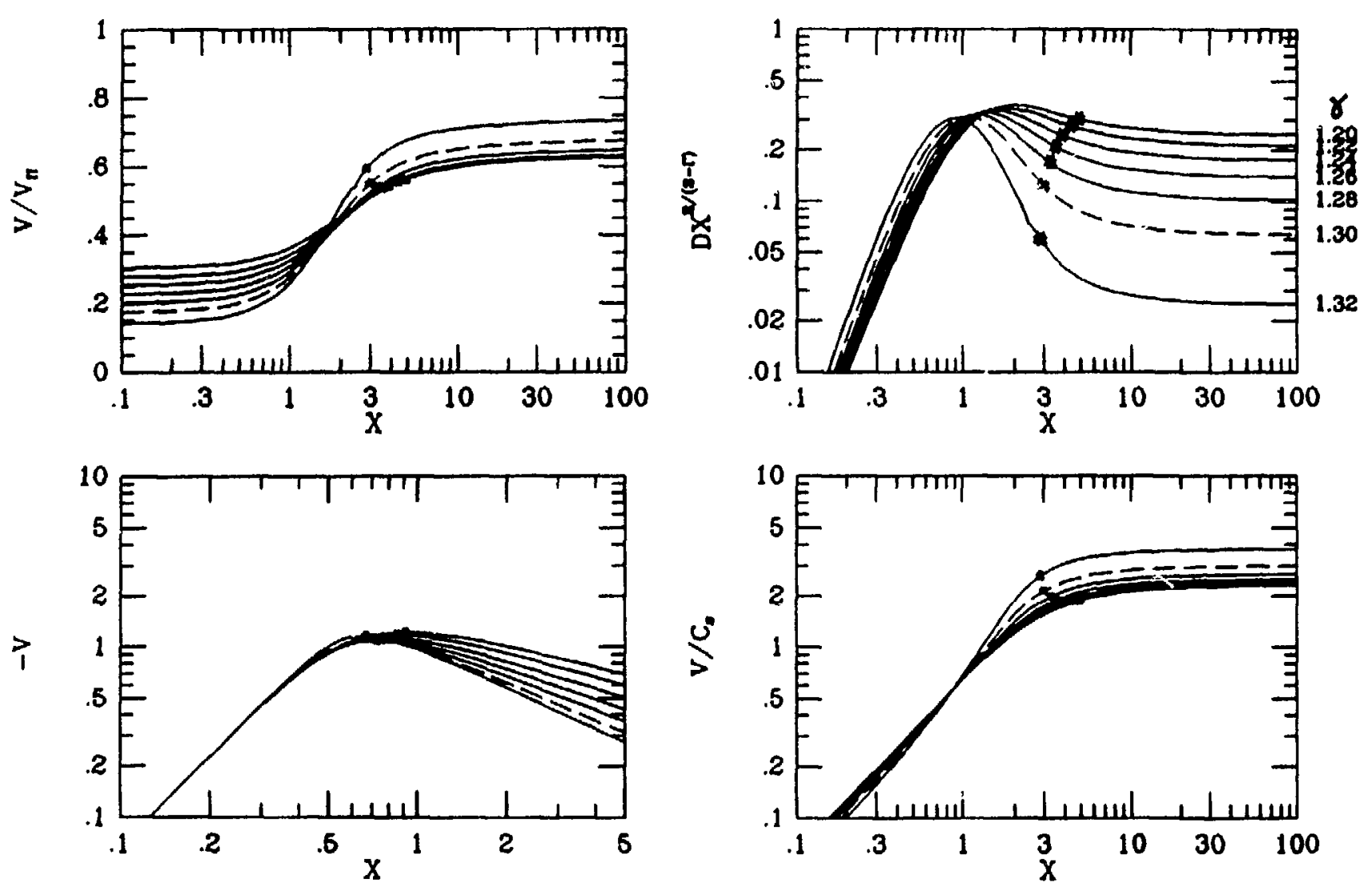

Fig. 6. Self-similar collapse. In $\gamma=1.20,1.22, \ldots, 1.32$, with $\gamma=1.30$ the dotted line, we give the Yahil solutions for $V / V_{\mathrm{ff}}, D X^{2 /(2-\gamma)},-V$, and $V / C_{s}$, as a function of $X$. The star gives the position of the maximum of infall velocity. $\gamma$ values are denoted alongside the top right figure. 
which is unphysical. (Note that $U$ and $A$ have the same asymptotic behavior so that $U / A \rightarrow$ constant as $X \rightarrow \infty$.) If $D_{0}$ is too large the derivatives become infinite at the sonic point. Thus, the correct $D_{0}$ is found and then the solution can be integrated numerically onward to $X \rightarrow \infty$.

Figure 6 shows the solutions of Yahil's equations for the seven cases $\gamma=$ $1.20,1.22, \ldots, 1.32$. The case $\gamma=1.30$, which has a special value being a good representation for supernova collapse, is is denoted with a dotted line. On each curve the starred point shows the position of the maximum velocity surface. We note the following: $-V / V_{f f}$ reaches an asymptotic value in the outer core, which drops with $\gamma$. Likewise, the Mach number $|V / A|$ reaches an asymptotic value which also decreases with $\gamma$. This has important consequences. It means that if $\gamma$ drops appreciably the outer core would be much less able to keep up with the collapsing inner core, which runs away.

The position of the maximum infall velocity surface moves outward somewhat as gamma drops, but the density distribution (shown by the combination $\left.D=X^{2 /(2-\gamma)}\right)$ remains higher for low $\gamma$. Note that the physical mass, $m \sim(-t)^{4-3 \gamma} M(X)$, so in the case of $\gamma$ appreciably less than $4 / 3$ the maximum velocity surface moves in quite a bit with time. Since this is where the shockwave will turn (actuaily at the sonic point which also moves inward) then this is harmful. For all these reasons Yahil claimed, "The difference $4 / 3-\gamma$ may be the most important parameter in determining success or failure."

An important quantity in the Yahil analysis is the ratio of the infall velocity to the freefall velocity at the center:

$$
\Omega_{0}^{-2}=\left.\left(\frac{v}{v_{f f}}\right)^{2}\right|_{r=0}=\left(6 \pi D_{0}\right)^{-1} \text {. }
$$

This is shown in figure 6 and is $(0.175)^{2}=0.0303$ for $\gamma=1.3,(0.226)^{2}=0.052$ for $y=1.26$ and will figure prominently in the next section. There we'll consider what happens when the central self-similarity solution breaks down, as the core bounces when the stiffness of nuclear matter produces a rapid increase in the adiabatic index.

\section{BOUNCE}

There is a lot of numerical experience with the process of bounce, but a good analytical theory is lacking. We will construct the first step of this, calculating the density at the maximum compression of the core, or "maximum scrunch." At first we will consider the simpler Newtonian case. We begin with the following observation of the numerical simulations. If we plot $v$ versus $r$ at two times, 


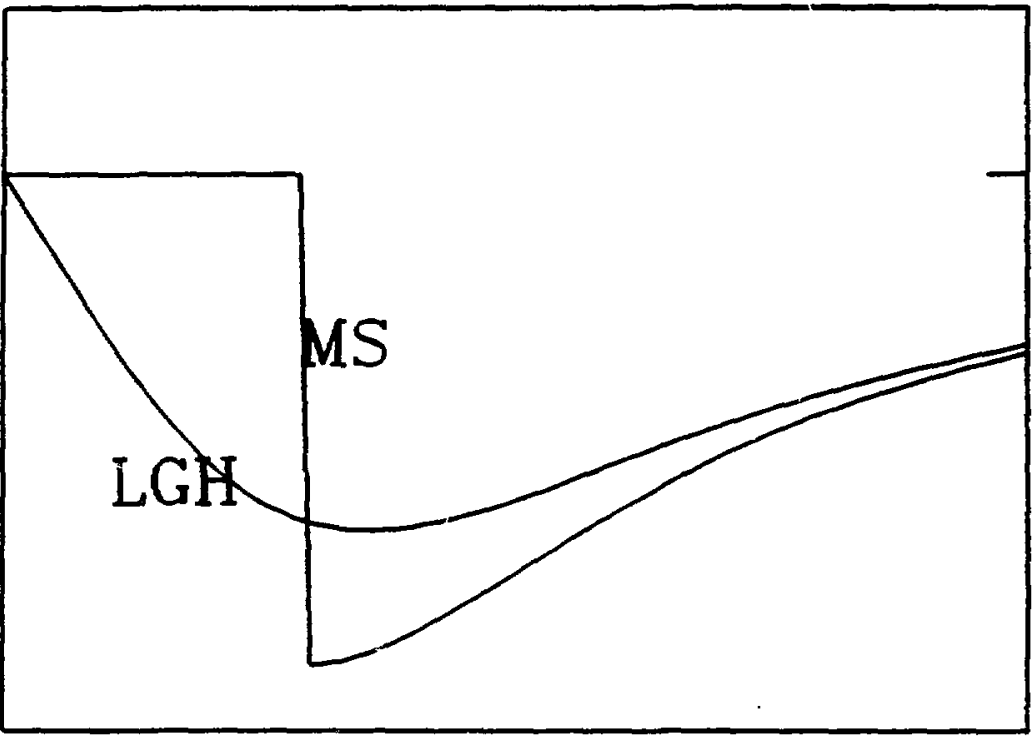

\section{Radius}

Fig. 7. Velocity profiles. $v$ vs $r$ is given schematically at two times, MS (maximum scrunch) and LGH (last good homology).

we shall call "last good homology" (LGH) and maximum scrunch (MS), we see something like figure 7 .

At either time, the velocity is proportional to radius in the central region, although at MS the constant of proportionality vanishes. This tempts us to propose that the velocity continuously levers in between these two times; i.e., that the flow is always homologous. This means that the radius of a given mass point $M$ can be writtein as

$$
r(M, t)=\alpha(t) r_{1}(M)
$$

where $r_{1}(M)$ is the radius of mass point $M$ when $\alpha=1$. Homologous flow must preserve $\rho r^{3}$ and thus

$$
\rho(M, t)=\alpha^{-3} \rho_{1}(M) .
$$

Since $\left.\frac{\partial \rho}{\partial r}\right|_{M=0}=0$ we can expand the density quadratically near the center

$$
\rho(M, t)=\rho_{c}(t)\left(1-\frac{r^{2}(M, t)}{a^{2}(t)}\right)=\rho_{c 1} \alpha^{-3}\left(1-\frac{r_{1}^{2}}{a_{1}^{2}}\right)
$$


where $\rho_{c}$ is the central density, since the polytropic radius $a(t)$ must foliow $a(t)=$ $\alpha a_{1}$ for the structure to be preserved. Near the center the Newtonian acceleration equation

$$
\frac{d v}{d t}+\frac{1}{\rho} \frac{\partial P}{\partial r}+\frac{G M}{r^{2}}=0
$$

becomes

$$
r_{1} \bar{\alpha}+c_{s}^{2}\left(-2 \frac{r_{1} \alpha}{a_{1}^{2} \alpha^{2}}\right)+\frac{4 \pi G}{3} \rho_{c 1} \alpha^{-3} r_{1} \alpha=0
$$

where we have used the Newtonian sound speed

$$
c_{s}^{2}=\frac{\partial P}{\partial \rho} \quad ; \quad \frac{\partial P}{\partial \tau}=c_{s}^{2} \frac{\partial \rho}{\partial r} .
$$

Thus, the acceleration equation can be rewritten as

$$
-\bar{\alpha} \alpha^{2}=\frac{4 \pi G}{3} \rho_{c 1}-2 \alpha \frac{c_{s}^{2}}{a_{1}^{2}} .
$$

We now define $\alpha=1$ to be where the acceleration vanishes and the core begins to decelerate; i.e.,

Then letting

$$
\left.\alpha\right|_{\bar{\alpha}=0}=1 .
$$

we find

$$
\dot{\alpha}_{1 \mathrm{ff}}^{2} \equiv \frac{8 \pi G}{3} \rho_{c 1}
$$

$$
a_{1}=2 c_{s 1} / \dot{\alpha}_{1 \mathrm{ff}},
$$

and we once again rewrite the acceleration equation as

$$
-\frac{1}{2} \ddot{\alpha} \alpha^{2}=\dot{\alpha}_{1 f f}^{2}\left(1-\alpha \frac{c_{s}^{2}}{c_{\delta 1}^{2}}\right) .
$$

Thus, when the acceleration vanishes, the polytropic radius divided by the freefall time equals the sound speed. This means the core passes through its hydrostatic equilibrium configuration, where perturbations are damped out by acoustical waves.

By multiplying by $\frac{\dot{\alpha} \alpha^{-2}}{\alpha_{1 \mathrm{f}}^{2}}$, this equation can be integrated. We get

$$
-\left(\frac{\dot{\alpha}^{2}-\dot{\alpha}_{1}^{2}}{\dot{\alpha}_{1 \mathrm{ff}}^{2}}\right)=-\left(\frac{1}{\alpha}-1\right)-\int_{1}^{\alpha} \frac{d \alpha}{\alpha} \frac{c_{s}^{2}}{c_{s 1}^{2}}
$$


The sound speed integral can be done exactly by noting

$$
\int \frac{d \alpha}{\alpha} c_{s}^{2}=\int \frac{d \alpha}{\alpha} \frac{d P}{d \rho}=-\frac{1}{3} \int \frac{d \rho}{\rho} \frac{d P}{d \rho}=-\frac{1}{3} \int \frac{d P}{\rho}=-\frac{1}{3}\left(\frac{\varepsilon+P}{\rho}\right)
$$

where we have used the thermodynamic identity

$$
\frac{d P}{\rho}=d\left(\frac{\varepsilon+P}{\rho}\right) \text {. }
$$

Thus,

$$
-\left(\frac{\dot{\alpha}^{2}-\dot{\alpha}_{1}^{2}}{\dot{\alpha}_{1 \mathrm{ff}}^{2}}\right)=-\left(\frac{1}{\alpha}-1\right)+\frac{1}{3 c_{s 1}^{2}}\left(\frac{\varepsilon+P}{\rho}\right)^{\alpha} .
$$

An important parameter in this problem is thus

$$
f \equiv \frac{\dot{\alpha}_{1}}{\dot{\alpha}_{1 \mathrm{ff}}}=\left(\frac{v}{v_{\text {ff }}}\right)_{\alpha=1}=\Omega_{0}^{-1 / 2},
$$

being the important quantity in the Yahil analysis of the previous section.

Denoting $\alpha_{\mathrm{MS}}$ as the scale parameter at maximum scrunch, when $\dot{\alpha}=0$, we find

$$
f^{2}=-\left(\frac{1-\alpha_{\mathrm{MS}}}{\alpha_{\mathrm{MS}}}\right)+\frac{1}{3 c_{s 1}^{2}}\left(\frac{\varepsilon+P}{\rho}\right)_{1}^{\alpha_{\mathrm{MS}}} .
$$

if we use a pure polytrope equation of state we find

so we would find

$$
\begin{aligned}
& c_{s}^{2}=\frac{\gamma P}{\rho} \quad ; \quad \frac{\varepsilon+P}{\rho}=\frac{\gamma}{\gamma-1} \frac{P}{\rho} \\
& \frac{P}{\rho}=\frac{P_{1}}{\rho_{1}} \alpha^{-3(\gamma-1)},
\end{aligned}
$$

$$
f^{2}=-\frac{\left(1-\alpha_{\mathrm{MS}}\right)}{\alpha_{\mathrm{MS}}}+\frac{1}{3(\gamma-1)}\left(\alpha_{\mathrm{MS}}^{-3(\gamma-1)}-1\right) .
$$

Note that if $\gamma=4 / 3,3(\gamma-1)=1$ so that the righthand side vanishes; i.e., there can be no halting the collapse. Thus, we must have $\gamma>4 / 3$ for the righthand side to be positive and for there to be a solution for $\alpha_{M S}$. This is just another indication that the gravitational and internal energies scale the same for a relativistic gas.

Suppose we take as a slightly more complicated EOS the BCK form

$$
P=\left(\frac{K_{e} U^{4 / 3}}{9 \cdot 4 / 3}+\frac{K_{0}}{9 \cdot \gamma}\left(U^{\gamma}-1\right)\right) \rho_{0},
$$

where $K_{e}$ includes both electrons and neutrinos, $U=\rho / \rho_{0}$, and $\rho_{0}$ is the density of saturated nuclear matter. The energy density is obtained by integration;

$$
\begin{aligned}
\frac{\varepsilon(U)}{\rho_{0} U} & =\frac{\varepsilon(U=1)}{\rho_{0}}+\int_{\rho_{0}}^{\rho_{0} U} \frac{d \rho}{\rho^{2}} P(\rho) \\
& =\frac{K_{e} U^{\cdot 1 / 3}}{9 \cdot 4 / 3 \cdot 1 / 3}+\frac{K_{0}}{9 \gamma}\left\{\frac{U^{\gamma-1}-1}{\gamma-1}+\left(\frac{1}{U}-1\right)\right\},
\end{aligned}
$$


since the nuclear pressure and energy vanish at $U=1$ (constant energies per particle won't concern us). Then

$$
\left(\frac{\varepsilon+P}{\rho}\right)=\frac{K_{e} U^{1 / 3}}{3}+\frac{K_{0}}{9(\gamma-1)}\left(U^{\gamma-1}-1\right) .
$$

If we now assume we can identify $\alpha=1$ with $U=1$; i.e., that deacceleration begins as soon as the matter passes through saturation density, we obtain

$$
U=\alpha^{-3}
$$

so that

$$
\left(\frac{\varepsilon+P}{\rho}\right)_{1}^{\alpha}=\frac{K_{e}}{3}\left(\frac{1-\alpha}{\alpha}\right)+\frac{K_{0}}{9(\gamma-1)}\left(\alpha^{-3(\gamma-1)}-1\right) .
$$

However, this is a simplification that we should remember, because numerical simulations show that $\bar{\alpha}=0$ when $U \sim 2 / 3$, because the matter undergoes the phase transition to uniform nuclear matter at about $2 / 3$ of the saturation density. But it helps considerably, if dishonestly.

Noting that

$$
c_{s 1}^{2}=\left.\frac{d P}{d \rho}\right|_{\alpha=U=1}=\frac{K_{e}}{9}+\frac{K_{0}}{9},
$$

we find after a little algebra

$$
-\frac{\left(\dot{\alpha}^{2}-\dot{\alpha}_{1}^{2}\right)}{\dot{\alpha}_{1 \mathrm{ff}}^{2}}=\frac{K_{0}}{K_{e}+K_{0}}\left\{-\left(\frac{1-\alpha}{\alpha}\right)+\frac{\left(\alpha^{-3(\gamma-1)}-1\right)}{3(\gamma-1)}\right\} .
$$

This is a rather beautiful equation because:

1) if $K_{0}=0$ it shows electrons alone cannot stop the collapse.

2) the dependence on the two nuclear parameters, $K_{0}$ and $\gamma$, is explicitly separated.

3) the same equation gives the behavior along the collapse trajectory (i.e., it gives $\dot{\alpha}$ ), or be setting $\dot{\alpha}=0$ it finds $\alpha_{M S}$, the maximum scrunch value. One can integrate to obtain $\alpha(t)$, but this is a messy, non-analytical integral.

To proceed further, we need to get some numbers, in particular $K_{e}, K_{0}$, and $f^{2}$. The leptonic pressure (assuming $T \sim 0$ ) comes from degenerate electrons and electron-neutrinos.

$$
\frac{P_{e}}{\rho}=\frac{K_{e} U^{1 / 3}}{9 \cdot 4 / 3}=\frac{\left(Y_{e} \varepsilon_{F_{e}}+Y_{\nu} \varepsilon_{F \nu}\right)}{4}
$$


Now $\varepsilon_{F e}=\left(3 \pi^{2} \rho Y_{e}\right)^{1 / 3} ; \varepsilon_{F \nu}=\left(2 \cdot 3 \pi^{2} \rho Y_{e}\right)^{1 / 3}$, the 2 coming in the neutrino case because they have only one helicity state. Thus

$$
K_{e}=\frac{9 \cdot 4 / 3}{4} U^{-1 / 3}\left(3 \pi^{2} \rho_{0} U\right)^{1 / 3}\left(Y_{e}^{4 / 3}+2^{1 / 3} Y_{\nu}^{4 / 3}\right) \hbar c .
$$

Now we will take $\rho_{0}=0.145 \mathrm{fm}^{-3}$, appropriate to neutron-rich matter with $Y_{e}=1 / 3$. Taking $Y_{e} \simeq 0.33, Y_{\nu}=0.07$, we can get an effective lepton number for the pressure and $K_{e}$ :

$$
\begin{aligned}
0.40 & =Y_{L}>Y_{\text {eff }}=\left(0.33^{1 / 3}+2^{1 / 3} 0.07^{4 / 3}\right)^{3 / 4}=0.37 \\
K_{e} & =255 \mathrm{MeV} .
\end{aligned}
$$

Now our $f^{2}=\Omega_{0}^{-1}$ of Yahil. Numerical simulations find $f^{2} \simeq(0.15)^{2}=$ 0.0225 ; i.e., the collapse proceeds at about $15 \%$ of free fall when the center passes into nuclear matter and the equation of state stiffens. This corresponds to Yahil's self-similar solutions for $\gamma \sim 1.31$ which would give $f \sim 0.16$. At any rate, we'll use $f=0.15$ for definiteness.

So we have at maximum scrunch

$$
f^{2}\left(1+K_{e} / K_{0}\right)=-\frac{\left(1-\alpha_{\mathrm{MS}}\right)}{\alpha_{\mathrm{MS}}}+\frac{\left(\alpha_{\mathrm{MS}}^{-3(\gamma-1)}-1\right)}{3(\gamma-1)} .
$$

Figire 8 shows the solutions for $\alpha_{M S}$ for $\gamma=2,2.5,3,3.5$, and 4.0 , each curve labeled by its value of $\gamma$. The horizontal dashed lines show what the solution would be if $f^{2}=(0.15)^{2}$ and $K_{0}=(0.1,0.2, \ldots 0.6) K_{e}$; i.e., the value of $\alpha_{M S}$ is where the curve for a specific $\gamma$ and $K_{0}$ cross. Thus, for $\gamma=2.5, K_{0}=153 \mathrm{MeV}$, collapse would be halted at $\alpha_{M S}=0.85$, corresponding to a density compression factor $U_{\min }=\alpha_{\min }^{-3}=1.63$. This is well borne out in numerical simulations (with Newtonian gravity!) Note that unless $K_{0}$ gets quite small, $\gamma$ is more important in determining the bounce central density.

In the next graph (Figure 9), $U_{\text {MS }}$ is displayed in the same fashion. The dashed lines show what happens if the collapse begins to halt at $U=1$; the solid lines if it deaccelerates at $U=2 / 3$. We can see that this would be catastrophic for our theory, so we'll ignore this for the moment.

Actual hydrodynamical simulations now demand the use of general relativistic dynamics, and to compare an analytical theory of bounce with them would be premature. However, it is possible to extend our analysis by linearizing the general relativistic dynamical equations and proceeding in a similar fashion. Although some of the simple scaling behavior of the $\gamma$ - and $K$-dependence is lost, the general features remain the same. In fact, I find that the analytical and numerical 


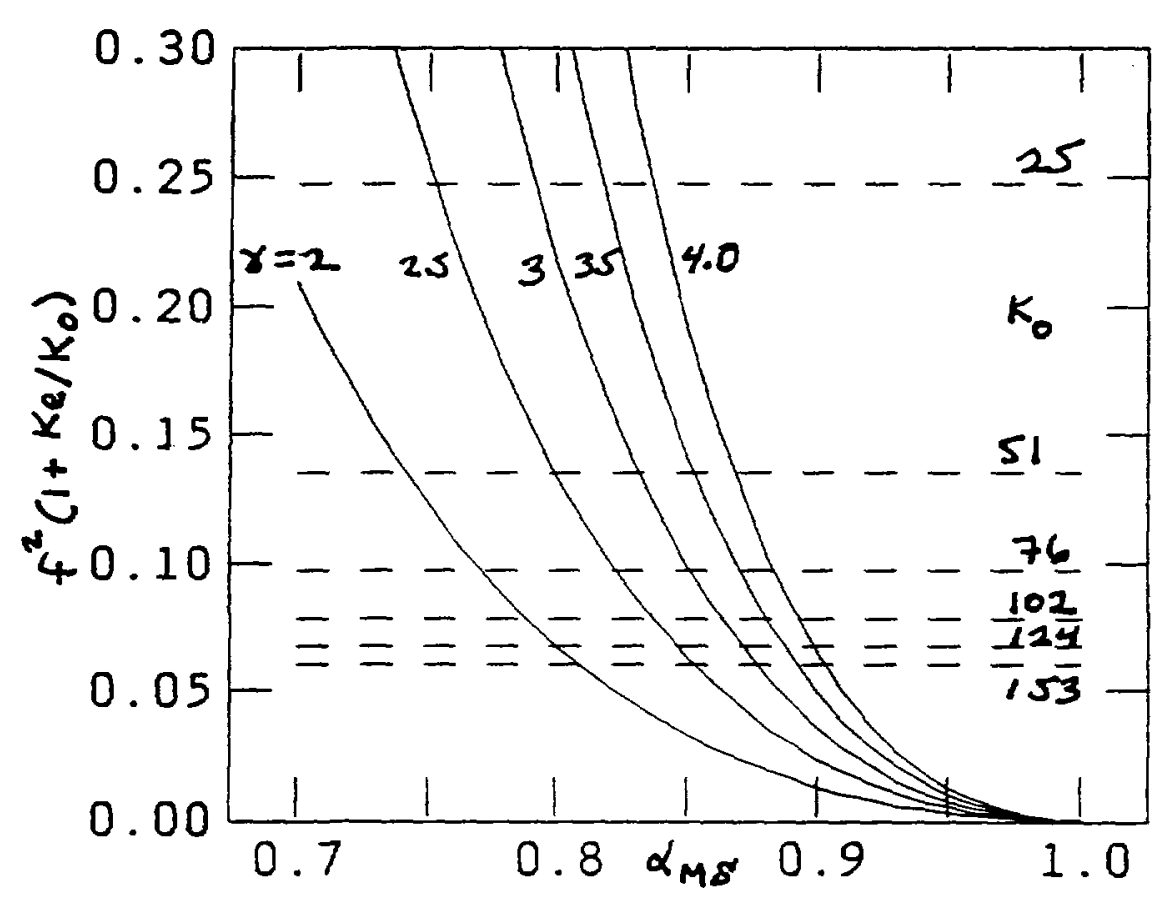

Fig. 8. Core compression amplitude, oMs, at maximum scrunch. The dashed lines give $\alpha_{\text {ms }}$ for the $K_{\mathrm{C}}$ 's listed at the right, assuming $K_{\mathrm{e}}=255 \mathrm{MeV}$.

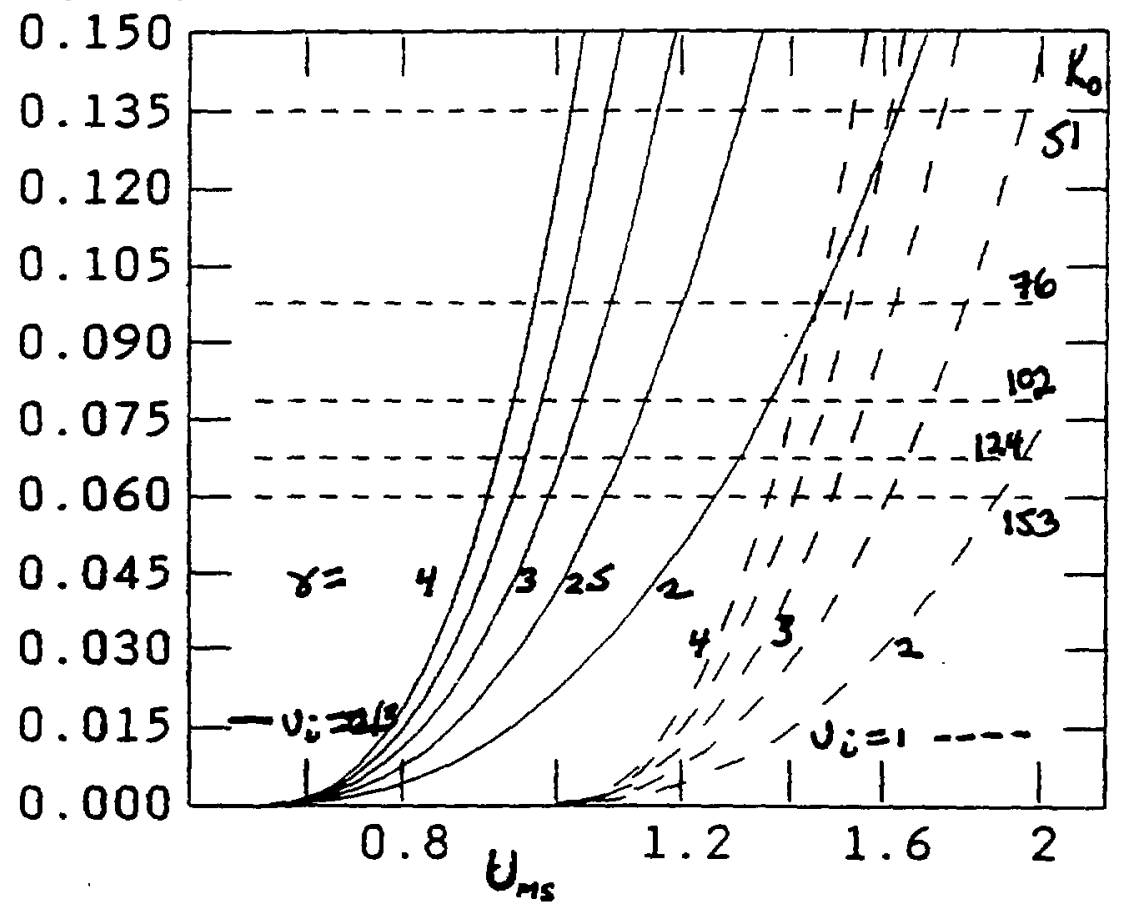

Fig. 9. Density at maximum scrunch. The dashed lines give $U_{m s}$ for the $K_{0}$ 's listed at the right, assuming $K_{e}=255 \mathrm{MeV}$. 
models compare even more favorably than in the Newtonian case. I had originally intended to continue in this direction for these lectures, but length of time does not permit an adequate treatment. At some point I hope to finish writing up this analysis and publish it.

We now have somewhat of a feel for how and when the core bounces. A far more difficult problem is to calculate the transfer of energy outward to the outer core of the star. To date, we have only numerical experience to guide us; a good analytical theory is completely lacking.

\section{EQUATION Of STATE}

First, we will consider the case of $\rho<\rho_{0}$, and utilize the compressible liquid drop model. We'll follow mostly Cooperstein (1985) and Bethe, Brown, Cooperstein, and Wilson (1983). Further references can be found in these papers.

\section{1. $\underline{T=0, \rho<\rho_{0}}$}

We write the energy per nucleon of nucleons as

$$
W=W_{\text {BULK }}+W_{\text {SIZE }}
$$

where $W_{\mathrm{BULK}}$ is the nuclear matter piece, and $W_{\text {SLEE }}$ the piece involving surface energies, Coulomb energies, etc., i.e., the things involving finite size. For the moment we ignore translational terms.

$W_{\text {BULK }}$ is easy to deal with. Here we are assuming that the density of the nucleonic matter is clustered near $\rho_{0}$, even though we intend $\rho \ll \rho_{0}$. So we write

$$
\begin{aligned}
& W_{\mathrm{BULK}}=W_{n m}+W_{\mathrm{s}}(1-2 x)^{2}+\frac{K_{0}(x)}{18}(1-\theta)^{2} \\
& W_{n m} \simeq-16 \mathrm{MeV}
\end{aligned}
$$

where $x=Z / A$, and $\theta=\rho_{0} / \rho_{\mathrm{s}}(x), \rho_{s}(x)$ being the saturation density at the given neutron excess, and $\rho_{0}$ is the density of the nuclear matter. $W_{\mathrm{BULK}}$ is taken as quadratic both in $(1 / 2-x)$, the neutron excess, and $(1-\theta)$ the density compression factor, since the energy of nuclear matter is minimized at saturation density. For $\theta \gg 1$ this is inadequate, and so our expression for high density uniform matter will be more complicated. The nuclear compression modulus

$$
K_{0}(x)=\left.9 \frac{\partial P}{\partial \rho}\right|_{\rho=\rho_{\theta}(x)}=\left.9 \frac{\rho^{2} \partial^{2} E}{\partial \rho^{2}}\right|_{\rho=\rho_{0}(x)}
$$

also has an $x$-dependence. Both $\rho_{s}$ and $K_{0}$ drop with $x$; the drop in $K_{0}$ coming mostly from the $\rho_{s}$, upon which it depends quadratically (see Kolehmainen et al, 


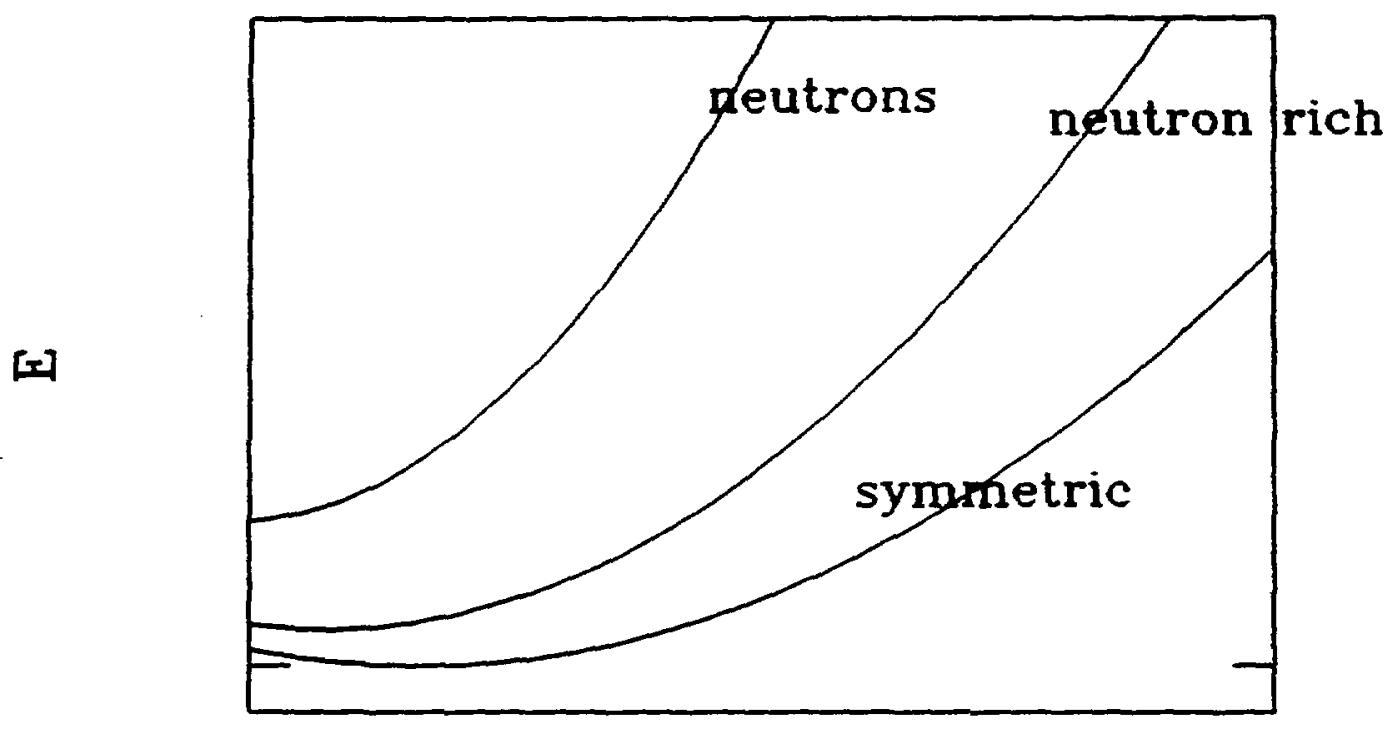

$\rho$

Fig. 10. Energy of nuclear matter. There is a minimum for symmetric matter and also one for neutron-rich matter, which is displaced to the top left. Neutron matter is unbound and has no minimum. A point of inflection is crossed when nuclear matter becomes so neutron-rich it is no longer bound, and $K_{0}$ vanishes.

1985). That $K_{0}$ must drop with $x$ is easy to see. If we graph $E(\rho)$ versus $\rho$ for different $x$, we get figure 10 .

So, since neutron matter is unbound, there must be a point of inflection where $K_{0}=0$; for $x<1 / 2 K_{0}$ drops until it vanishes, and $\rho_{s}(x)$ should drop also. How do $K_{0}$ and $\rho_{0}$ drop with $x$ ? They are both quadratic in $(1-2 x)$, and with $S K M^{*}$ parameters you would get

$$
\begin{aligned}
& \rho_{s}(x)=\rho_{s}(1 / 2)\left(1-\frac{3}{4}(1-2 x)^{2}\right) \\
& K_{0}(x)=K_{0}(1 / 2)\left(1-2(1-2 x)^{2}\right)
\end{aligned}
$$

where $\rho_{\text {. }}(1 / 2) \simeq 0.16 \mathrm{fm}^{-3}\left(=2.7 \cdot 10^{14} \mathrm{gm} / \mathrm{cm}^{3}\right)$, and $K_{0}(1 / 2) \sim 210 \pm 30 \mathrm{MeV}$ from Giant Monopole Resonance analysis of the breathing mode. BCK like to use $K_{0}(1 / 2)=180$. With this choice

$$
\begin{aligned}
& K_{0}(1 / 3)=140 \mathrm{MeV} \\
& \rho_{s}(1 / 3)=0.145 \mathrm{fm}^{-3}
\end{aligned}
$$


These quadratic expressions shouldn't be used for very small $x$. However, an improvement would be

$$
\rho_{s}(x)=\frac{\rho_{x}(1 / 2)}{\left(1+3 / 4(1-2 x)^{2}\right)}
$$

and a similar expression for $K_{0}(x)$, which have the same behavior near $x=1 / 2$.

The size energy now depends on the fraction of the total volume occupied by the nuclear phase. This is the "packing fraction,"

$$
u \equiv \rho_{0} / \rho,
$$

where $\rho$ is the total baryon density. We write

$$
W_{\mathrm{SIZE}}=\frac{\left[W_{\mathrm{surf}} A^{2 / 3}+W_{\mathrm{coul}} A^{5 / 3}\right]}{A}
$$

in the case of a nucleus. We haven't included curvature terms, which are poorly known, and which tend to be cancelled by translational terms we also neglect (see Cooperstein and Wambach, 1984). Since $A \sim R^{1 / 3}, 4 \pi R^{2} \sim A^{2 / 3} ; Z^{2} / R \sim$ $A^{2} / A^{1 / 3} \sim A^{5 / 3}$ giving the $A$ dependence of the surface energies and coulomb energies. If we assume all dependence on $x$ and $u$ is in $W_{\text {surf }}$ and $W_{\text {coul }}$, then we

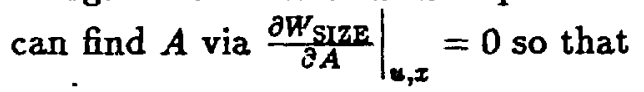

$$
A=\frac{1}{2} \frac{W_{\text {surf }}}{W_{\text {coul }}}
$$

Then

$$
W_{\text {SIZE }}=\frac{3}{2} W_{\text {surf }} A^{-1 / 3}=3 W_{\text {coul }} A^{2 / 3}=3\left(\frac{W_{\text {surf }}^{2} W_{\text {coul }}}{4}\right)^{1 / 3} .
$$

As $A$ increases, the surface energy per nucleon goes down because less nucleons are on the surface, while the coulomb energy still goes up. The result is the above virial theoreom, which says

$$
\text { coulomb energy }=1 / 2 \text { surface energy }
$$

The surface energy can be written as

$$
E_{\text {surf }}=W_{\text {earf }} A^{-1 / 3}=\frac{1}{A} 4 \pi R^{2} \sigma_{\text {aurf }}(x) .
$$

If we assume $R=R_{0}\left(\frac{\rho_{0}}{0.16}\right)^{-1 / 3} A^{1 / 3}$, we get

$$
W_{\text {surf }}=4 \pi\left(\frac{0.16}{\rho_{0}}\right)^{2 / 3} R_{0}^{2} \sigma_{\text {surf }}(x)
$$


In doing so we have assumed that the neutron excess $(1 / 2-x)$ is constant through the space inhabited by nucleons. In fact things are more complicated, and there is a "neutron skin," but we will neglect this. Taking $R_{0}=1.14 \mathrm{fm}$; $\sigma_{s}(x)=17.8 x^{2}(1-x)^{2} \mathrm{MeV} / \mathrm{fm}^{2}$, we get

$$
\begin{aligned}
W_{\text {surf }} & =290 x^{2}(1-x)^{2}\left(\frac{0.16}{\rho_{0}}\right)^{2 / 3} \mathrm{MeV} \\
& =\frac{290 x^{2}(1-x)^{2}}{\left(\theta\left(1-\frac{3}{4}(1-2 x)^{2}\right)\right)^{2 / 3}}
\end{aligned}
$$

Note that for $x=1 / 2, \sigma_{s}=\frac{17.8}{16}=1.1 \mathrm{Mev} / \mathrm{fm}^{2}$ is the surface tension energy.

To calculate the Coulomb energy we consider an electrically neutral "WignerSeitz" cell with a nucleus at the center, displayed in figure 11 .
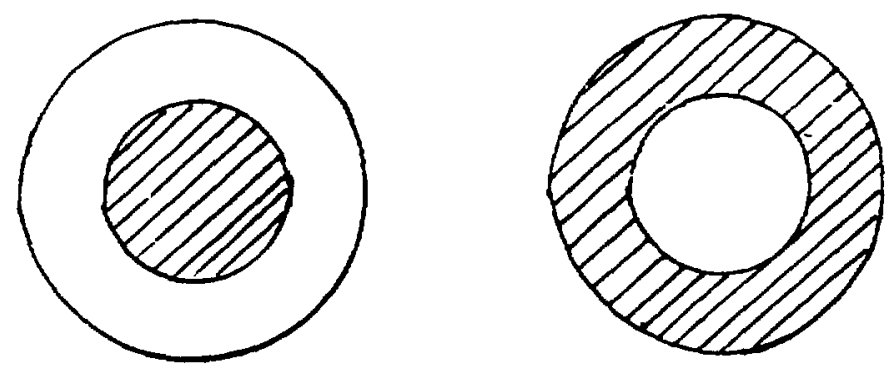
a (nuclei)
b (bubbles)

Fig. 11. Wigner-Seitz cells: The dense matter is in the hatched regions.

Now, $\rho=\frac{A}{(4 \pi / 3) R_{c}^{3}} ; \quad \rho_{0}=\frac{A}{(4 \pi / 3) R^{3}}$, so $u=\rho / \rho_{0}=\left(R / R_{c}\right)^{3}$ is the fraction of the volume inside the nucleus. To calculate the electrostatic configuration, we note that if there were no electrons in the cell we would have as the electrostatic energy of a uniform sphere

$$
E_{\mathrm{coul}}=\frac{1}{A} \frac{3}{5} \frac{Z^{2} e^{2}}{R}
$$

But the cell is electrically neutral, and we assume (not too bad an approximation) that the electrons uniformly inhabit the cell; they go in and out of the nucleus at will. Then the charge inside the nucleus is 


$$
W_{\text {SIZE }}(x, \theta, u)=\beta(x) \theta^{-1 / 3} G(u)
$$

where

$$
\beta(x)=\frac{75.4 x^{2}(1-x)^{4 / 3}}{\left(1-\frac{3}{4}(1-2 x)^{2}\right)^{1 / 3}} .
$$

This is very convenient because the $x, \theta$, and $u$ dependences are factorized.

\section{2. $\quad$ Bubbles}

Suppose, as in figure $11 \mathrm{~b}$, we invert the geometry, putting the matter outside bubbles. If the matter were to turn "inside out" when $u=1 / 2$, then the amount of surface area would be the same, and it is clear that for $u>1 / 2$ energy would be better minimized by shrinking a bubble rather than filling up the Wigner-Seitz cell at its outer boundary. It is possible to calculate $W_{\text {SIZE }}$ in this bubble phase by reinterpreting the nucleus case. All we have to do is make the substitution that

$$
\begin{aligned}
G_{\mathrm{bub}}(u) & =\frac{(1-u)}{u} G_{\mathrm{nuc}}(1-u)=\frac{1-u}{u} g^{1 / 3}(1-u) \\
& =\frac{1-u}{u}\left(1-\frac{3}{2}(1-u)^{1 / 3}+\frac{1}{2}(1-u)\right)^{1 / 3}
\end{aligned}
$$

(See Bethe et al 1983.)

\section{HOMEWORK}

Convince yourself it is true $G_{\text {bub }}=\frac{(1-u)}{u} G_{\text {nuc }}(1-u)$ by calculating $G_{\text {bab }}(u)$ directly.

Note there is an important difference between the bubble and nuclear phases. In either phase the coulomb energy wants the nuclear phase to fill the entire cell so that the electrically neutral system has a vanishing electrostatic energy. But the surface tension in the nuclear case fights this, by compressing the nucleus; in the bubble case the surface tension tries to collapse the bubble to eliminate the surface; thus the bubble may collapse unless $K_{0}$, which resists this expansion of the nuclear matter phase, is large.

In either case we have

$$
W=W(x, \theta, u) \quad ; \quad \rho=\rho_{0} u=\rho_{d}(x) \theta u
$$


Thus, at fixed $x$

$$
\begin{aligned}
d \ln \rho & =d \ln \theta+d \ln u \\
\frac{P}{\rho} & =\left.\frac{d W}{d \ln \rho}\right|_{x}=\left.\frac{\partial W}{\partial \ln \theta}\right|_{x, u} \frac{d \ln \theta}{d \ln \rho}+\left.\frac{\partial W}{\partial \ln u}\right|_{x, \theta} \frac{d \ln u}{d \ln \rho} \\
\frac{P}{\rho} & =\left.\frac{\partial W}{d \ln \theta}\right|_{x, u}+\left[\left(\frac{\partial W}{\partial \ln u}\right)_{x, \theta}-\left.\frac{\partial W}{\partial \ln \theta}\right|_{x, u}\right] \mid \frac{d \ln u}{d \ln \rho}
\end{aligned}
$$

Now at fixed $\ln \rho, \delta W$ must vanish. Thus we obtain for the pressure per particle

$$
\frac{P}{\rho}=\left.\frac{\partial W}{\partial \ln \theta}\right|_{x, u}=\left.\frac{\partial W}{d \ln u}\right|_{x, \theta} .
$$

To see how the system determines $\theta$ and $u$ given $\rho$ and $x$, we evaluate the above equilibrium constraint. Thus,

$$
\begin{aligned}
\frac{\partial W}{\partial \ln \theta} & =\frac{\partial W_{\mathrm{SIZE}}}{\partial \ln \theta}+\frac{\partial W_{\mathrm{BULK}}}{\partial \ln \theta}=-\frac{1}{3} W_{\mathrm{SIZE}}-\frac{K_{0}}{9} \theta(1-\theta) \\
\frac{\partial W}{\partial \ln u} & =W_{\mathrm{SIZE}} \frac{G^{\prime}}{G} .
\end{aligned}
$$

So

or

$$
W_{\text {SIZE }}\left(\frac{1}{3}+\frac{G^{\prime}}{G}\right)+\frac{K_{0}}{9} \theta(1-\theta)=0
$$

$$
\theta^{4 / 3}(1-\theta)+\varepsilon_{\text {sise }}\left(\frac{G}{3}+G^{\prime}\right)=0
$$

(see figure 12). Thus given $x$, this equation determines $\theta$ vs $u$; and then $\rho=$ $\rho_{s}(x) \theta \cdot u$ is obtained. It depends only on

$$
\frac{\text { size energy of isolated nucleus }}{\text { compression modulus }}=\varepsilon_{\text {size }}=\frac{9 \beta(x)}{K_{0}(x)}
$$

which is the basic parameter in the problem. In the case of iron, we'd have $\varepsilon_{\text {sixe }} \simeq 9 \times 7 / K_{0} \sim 0.25$.

Some special cases can be easily derived and are listed in table 2.

Table 2 -- Linits on $\theta$, the nuclear compression

\begin{tabular}{|c|c|c|}
\hline$u$ & $\frac{G}{3}+G^{\prime}$ & $\theta$ \\
\hline \hline 0 & $1 / 3 \cdots$ & $\sim 1+\frac{1}{3} \varepsilon_{\text {aise }} \simeq 1.08$ \\
\hline 1 & -1 & $\sim 1-\varepsilon_{\text {aize }} \simeq 0.75$ \\
\hline $1 / 4$ & $\sim 0$ & $\sim 1$ \\
\hline
\end{tabular}




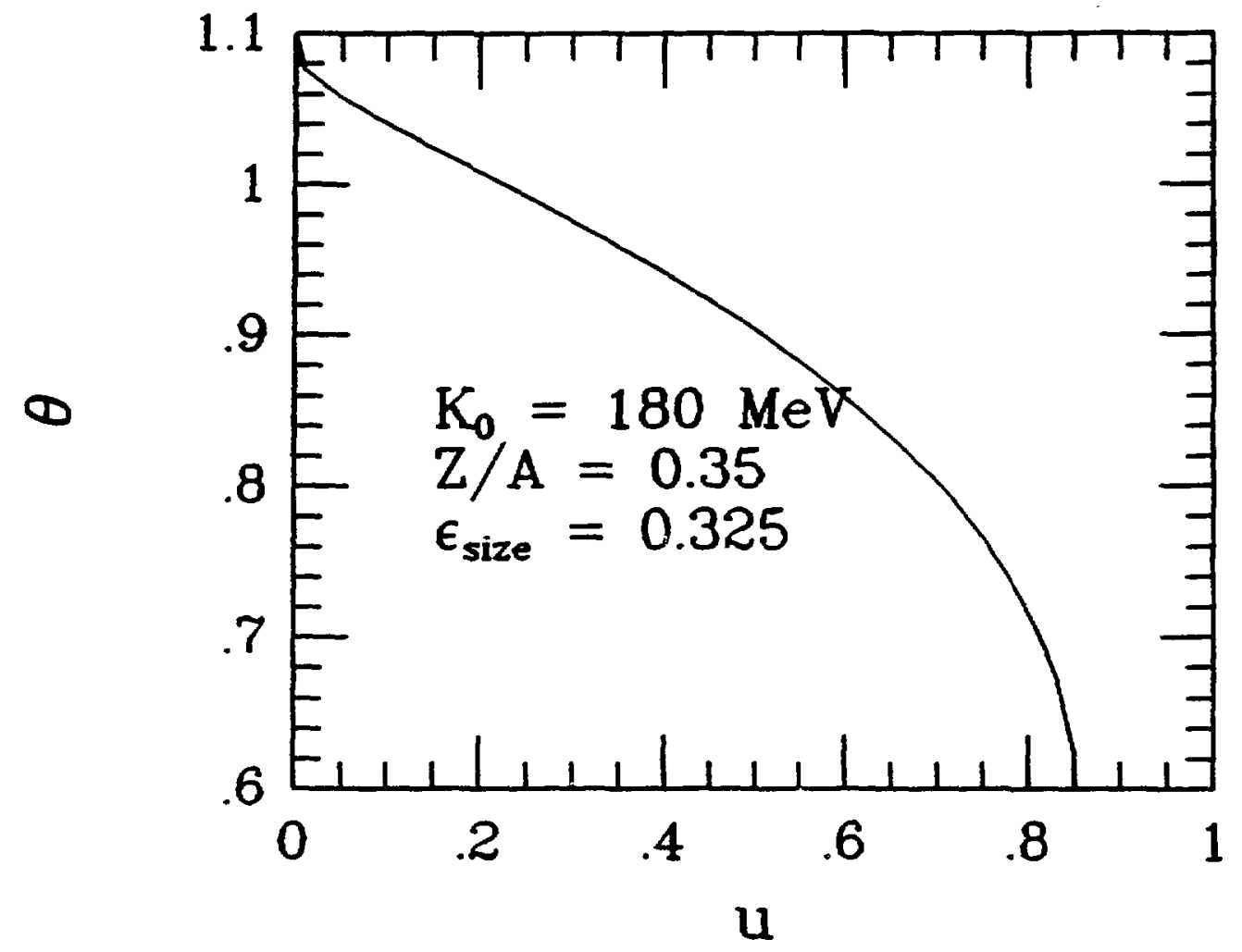

Fig. 12. Packing fraction and nuclear compression.

For $u \ll 1$ :

$$
\begin{aligned}
& G(u)=G_{\text {nuc }}(u) \simeq\left(1-3 / 2 u^{1 / 3}\right)^{1 / 3} \simeq 1-\frac{u^{1 / 3}}{2} \\
& G^{\prime} \simeq-\frac{1}{6} u^{1 / 3} \\
& \theta^{4 / 3}(1-\theta)+\varepsilon_{\text {nize }}\left(\frac{1}{3}+\frac{1}{6} u^{1 / 3}-\frac{1}{6} u^{1 / 3}\right)=0
\end{aligned}
$$

or $\theta^{4 / 3}(1-\theta) \simeq-\varepsilon_{\text {airo }} / 3$; or $\theta \simeq 1+\varepsilon_{\text {iize }} / 3$. Thus for $u \rightarrow 0$ from figure 13 , we see $\varepsilon_{\text {sire }} \sim 0.25$ for symmetric matter, so $\theta \sim 1.08$. Thus the nucleus is compressed by $\sim 8 \%$ over saturation due to the surface tension winning out over the coulomb force.

At $u=0.25$, we find $\theta=1$ independent of $\varepsilon_{\text {size; }}$ i.e., at a packing fraction of one fourth, the nuclear matter phase is at saturation density. 
For $u \sim 1$,

$$
\begin{aligned}
& G \simeq \frac{(1-u)}{u}\left(1-\frac{3}{2}(1-u)^{1 / 3}+\frac{(1-u)}{2}\right)^{1 / 3} \simeq(1-u) \\
& \frac{G}{3}+G^{\prime} \simeq \frac{1-u}{3}-u=\frac{(1-4 u)}{3} \simeq-1 .
\end{aligned}
$$

Thus

$$
\theta^{4 / 3}(1-\theta) \simeq-\frac{\varepsilon_{\text {sire }}}{3}(1-4 u) \simeq+\varepsilon_{\text {size }} ; \theta \simeq 1-\varepsilon_{\text {size }} ; u \rightarrow 1
$$

i.e., the bubble disappears when $\theta<1 ; \theta \sim 0.75,0.8$ for $\varepsilon_{\text {size }} \sim 0.25,0.20$. Thus we go to uniform nuclear matter at $\rho \sim 0.8 \rho_{s}(x)$ while the nuclear pressure is still negative, a condition known as "cavitation." How does the nucleus-bubbles transition go?

For a first order phase transition we should have equality of the quantities, $P$ and $\mu$. At $T=0, \mu=E+P / \rho$, thus

$$
\begin{aligned}
& \mu_{\text {nuc }}=\mu_{\text {bub }}=E_{\text {nuc }}+\frac{P_{\text {nuc }}}{\rho_{\text {nuc }}}=E_{\text {bub }}+\frac{P_{\text {bub }}}{\rho_{\text {bub }}} \\
& P_{\text {auc }}=P_{\text {bub }} \\
& \left(E_{\text {bub }}-E_{\text {nuc }}\right)=-P\left(\frac{1}{\rho_{\text {bub }}}-\frac{1}{\rho_{\text {nuc }}}\right)
\end{aligned}
$$

(Electrons must be included to obtain meaningful results here!) Near $u=1 / 2, E_{\text {bub }}=$ $E_{\text {nuc }}$, so we don't expect a large discontinuity in density. It takes a considerable amount of algebra (or a small amount of numerics!) to show that the discontinuity is indeed small near the transition. In addition, we should remember that the leptonic pressure (electrons and neutrinos) is far greater than the nucleonic piece in supernova matter, and this makes the effects of the phase transition weaker. In fact, without the presence of the electrons, there would be no transition, as otherwise the total pressure would be negative for both nucleonic phases, and each would be unstable.

Now it is possible to have a whole series of other geometries besides nucleii and bubbles, in order of symmetries; these are displayed in figure 13.

Such geometries were calculated by Ravenhall, Pethick and Wilson, (1983) and also by Williams and Koonin. But the energy differences of these phases are small (keV), and at $T \sim$ several $\mathrm{MeV}$ we don't really care - the true situation is to expect a "casserole" of different shapes.

My solution was to adopt the following formula:

$$
\begin{aligned}
G(u) & =(1-u) G_{\mathrm{nuc}}(u)+u G_{\mathrm{bab}}(u) \\
& =(1-u)\left[g^{1 / 3}(u)+g^{1 / 3}(1-u)\right]
\end{aligned}
$$




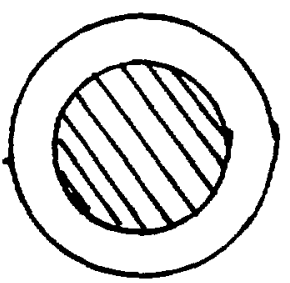

nuclei
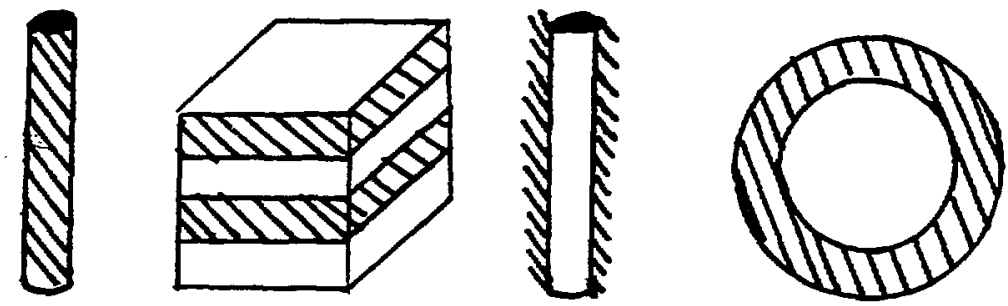

bubbles

Fig. 13. Subnuciear-density phases.

which is graphed in figure 14, along with the solution of Ravenhall et al,

$$
G_{\mathrm{RPW}}=\left[\frac{5}{9} \frac{d^{2}}{d+2}\left[\frac{1-\mathrm{d} / \mathrm{2}^{1-2 / d}}{d-2}+\frac{u}{2}\right]\right]^{1 / 3}
$$

where $d$ is the dimension of the symmetry. (The bubble phases are obtained by $G_{\text {bub }}=\frac{1-u}{u} G_{\text {nuc }}$ )

It's clear my formula smoothly passes along all the geometries. The physical content is: the size energy should depend only on the total surface area of the system, and this should be a smooth function of density (actually $u$, the packing fraction).

\section{3. $T>0$ : Effective Mass and Drip Vapor}

At finite temperatures, the picture changes considerably. My 1985 Nuclear Physics article considers this situation. Fortunately, an improvement is that things behave more smoothly due to the additional flexibility afforded by the increase in the degrees of freedom available. However, we pay a price of additional complexity. There are essentially two new degrees of freedom. The first is the population of excited intrinsic nuclear states. The second is the appearance of a nuclear statistical equilibrium mixture of species, with protons and neutrons dripping out of the condensed phase to be free, and a variety of nuclear species now existing, including light fragments such as alpha particles.

Instead of the energy we must now work with the free energy, $F=E-T S$. The entropy is obtained as

$$
S=\frac{-\partial F}{\partial T} .
$$




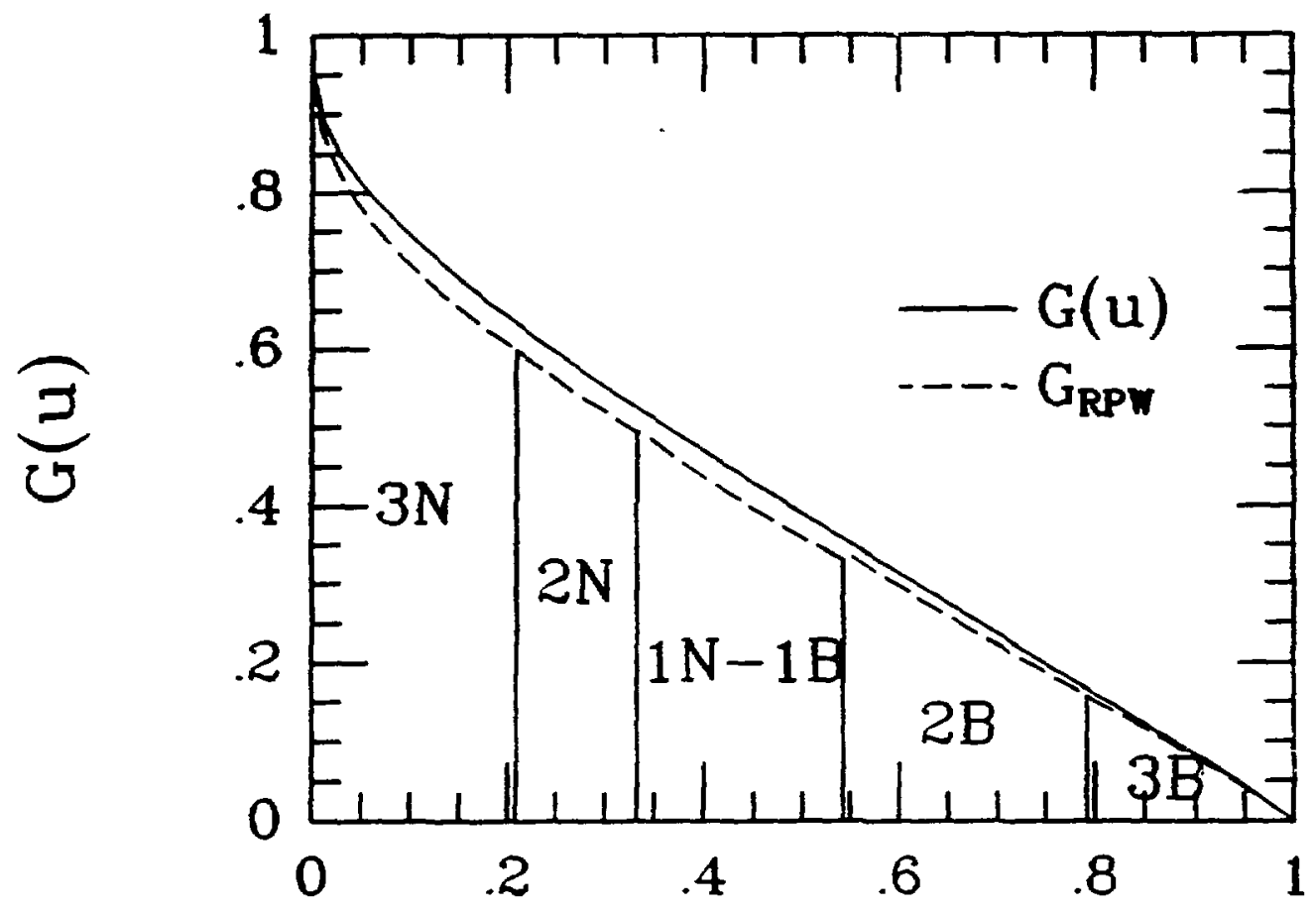

u

Fig. 14. Geometric function $G(u)$. Solid line gives $G(u)$, my geometric function, and the dashed line the solution for the energetically favored phases of Ravenhall, Pethick and Wilson, $G_{\mathrm{RPW}}(u)$. The regions labelled are: $3 N$ (nuclei), $2 N$ (nuclear spaghetti), $1 N-1 B$ (lasagne and anti-lasagne, which are the same), $2 B$ (anti-spaghetti), and $3 B$ (bubbles).

Before including "drip" or translational terms, we now add an excited state contribution to the free energy $B$ :

$$
\begin{aligned}
& B=F=W_{\mathrm{BULK}}+W_{\mathrm{SIZE}}-\frac{a}{A} \frac{m^{*}}{m} T^{2} \\
& \frac{a}{A}=\frac{\pi^{2}}{4 E_{f}}=(\theta \phi)^{-2 / 3}(14.9)^{-1} \mathrm{MeV} \quad\left(\theta \phi=\frac{\rho_{0}}{0.16}\right) \\
& \frac{m^{*}}{m}=m_{s}+\left(m_{0}-m_{s}\right) \frac{W_{\mathrm{SIZE}}}{W_{\mathrm{SIZE}}\left[\mathrm{Fe}^{56}\right]=6.88 \mathrm{MeV}} .
\end{aligned}
$$

$E_{f}$ is the nucleon fermi energy. The idea here is that the additional density of states due to the surface is proportional to the size energy and can be parametrized in the effective mass. In our expression for the effective mass, $m_{0}=2$ 
is $m^{*} / m$ in the iron peak, and $m_{s}=0.7$ is $m^{*} / m$ for saturated matter. This treatment will only be good below the critical point of the liquid vapor transition; near this more has to be done. With the energy dependence of $m^{*}$ not yet included, this leads to

$$
\frac{P}{\rho} \simeq \beta \theta^{-1 / 3} G \cdot\left[1-(T / 9)^{2}\right] .
$$

This is very important, because this thermal pressure arising from the reduction of the effective mass with increasing density will raise $\Gamma$ before $\rho=\rho_{s}$, when the star collapses.

If we include drip, we now have a four-component mixture: $n, \rho, \alpha$ and a heavy nucleus, $H$, with $(Z, A)$. Four constraints must now be satisfied in addition to conservation of charge and mass:

$$
\begin{aligned}
& \frac{\mu_{\alpha}}{4}=\mu_{n}-1 / 2 \hat{\mu} \\
& B+\frac{\mu_{H}^{\mathrm{tr}}}{A}+\frac{\partial B}{\partial \log \theta}=\mu_{n}-x \hat{\mu} \\
& \frac{\partial B}{\partial x}+\left(\frac{\partial B}{\partial \log \phi}-\frac{\partial B}{\partial \log \theta}\right) \frac{\partial \log \phi}{\partial x}=-\hat{\mu} \\
& \frac{\partial B}{\partial \log \theta}-\frac{\partial B}{\partial \log u}=\frac{Y T}{1-u} \frac{\rho}{\rho_{0}},
\end{aligned}
$$

where $Y \equiv X_{n}+X_{p}+1 / 4 X_{\alpha}+1 / A X_{H}$. The last equation is the pressure balance at surface, and

$$
\frac{P}{\rho}=\frac{Y T}{1-u}+X_{H} \frac{\partial B}{\partial \log u}+Y T+\frac{\partial B}{\partial \log \theta} X_{H}
$$

is the nuclear contribution to the pressure.

Some important new behavior follows. At $T>0$ nucleons (mostly neutrons) "drip" out of the dense phase. Thus $Z / A>Y_{e}$. This feedback drastically alters behavior along adiabats by increasing the surface tension, etc. It turns out that the thermal effects (and the fact that $Z / A<0.5$ ) tend to be larger than many $T=0$ phenomena.

\section{NEUTRON STARS}

Here the written notes will be terse. A fuller explanation of general neutron star properties can be found in my 1988 Phys. Rev. C article, and a discussion of strange matter can be found in Bethe, Brown, and Cooperstein (1987) and in my contribution to the Windsurfing the Fermi Sea vol. 2, Coopenstein (1987). I have chosen not to write up the strange matter material, as my 1987 article is a sufficient review of our theory. 
The basic equations of neutron star structure are the hydrostatic relations,

$$
\begin{aligned}
\frac{d M}{d r} & =4 \pi r^{2} \varepsilon \\
\frac{-d P}{d r} & =\frac{G\left(M+4 \pi r^{3}\right)(\varepsilon+P)}{r^{2}\left(1-\frac{2 G M}{r}\right)},
\end{aligned}
$$

where $P, \varepsilon$, and $\rho$ are the pressure, energy density, and baryon density, $M$ the gravitational mass, and we have taken $c=1$. These equations are closed when we specify the functional relation of $P, \varepsilon$, and $\rho$. Then the equation can be integrated numerically by standard means, and the edge of the star comes when the pressure vanishes. However, if we can use a simplified EOS, as Bludman (1979) did, then we can get pretty far analytically, and restrict the number of models we need analyse. If we use the relativistic polytropic equation of state

$$
P \sim \varepsilon^{\Gamma}
$$

we get the dimensionless equations (Cooperstein 1988)

$$
\begin{aligned}
& \frac{d m}{d z}=z^{2} \theta^{n} \\
& \frac{\partial \theta}{\partial z}+\frac{\left(m+z^{3} \theta^{n+1} \sigma_{c}\right)\left(1+\sigma_{c} \theta_{c}\right)}{z^{2}\left(1-2(n+1) \sigma_{c} m / z\right)}=0,
\end{aligned}
$$

where the relativistic generalizations of the usual polytropic quantities are

$$
\begin{aligned}
& \Gamma=1+1 / r \\
& r=a z \\
& a^{2}=\frac{(n+1) \sigma_{c}}{4 \pi G \varepsilon_{c}} \\
& M=4 \pi a^{4} \varepsilon_{c} \cdot m \\
& P=P_{c} \theta^{n+1} \\
& \varepsilon=\varepsilon_{c} \theta^{n} \\
& \sigma=P / \varepsilon=\sigma_{c} \theta
\end{aligned}
$$

In dimensional variables,

$$
\begin{aligned}
& M=\frac{(n+1)^{3 / 2} \sigma_{0}^{n / 2}}{G\left(4 \pi G \varepsilon_{0}\right)^{1 / 2}} \widetilde{M} \\
& R^{2}=a^{2} z_{1}^{2}=\left(\frac{n+1}{4 \pi G}\right) \frac{\sigma_{0}^{n}}{\varepsilon_{o}} \sigma_{c}^{1 \sim n} .
\end{aligned}
$$

where the subscribed zero indicates evaluation at $\rho_{0}$, and 


$$
\begin{aligned}
& m_{1}=\frac{\left(-z_{1}^{2} \theta_{1}^{1}\right)}{1+2(n+1)\left(-z_{1}^{2} \theta_{1}^{\prime}\right) \sigma_{c}} \\
& \widetilde{M}=\sigma_{c}^{\frac{3-n}{2}} m_{1}
\end{aligned}
$$

Thus all the dependence for a given value of $n$ is centered in $\widetilde{M}$, which can be obtained numerically. At saturation density, the relativistic effects are small, and thus

$$
\begin{aligned}
& \varepsilon_{0} \cong \rho_{0} m_{N}=150 \mathrm{MeVfm}^{-3} \\
& P_{0}=\frac{K_{0} \rho_{0}}{9 \Gamma} \\
& \sigma_{0}=\frac{F_{0}}{\varepsilon_{0}}=\frac{K_{0}}{9 \Gamma m_{N}}=\frac{K_{0} n}{9 m_{N}(n+1)} .
\end{aligned}
$$

Now, putting in numbers,

$$
\begin{aligned}
& M=13.5 M_{\odot} \cdot(n+1)^{3 / 2}\left[\frac{n}{n+1} \frac{K_{0}}{9 m_{N}}\right]^{n / 2} \cdot \widetilde{M} \\
& R^{2}=(20.1 \mathrm{~km})^{2} \cdot(n+1)\left[\frac{n}{n+1} \frac{K_{0}}{9 m_{N}}\right]^{n} \cdot \sigma_{c}^{1-n}
\end{aligned}
$$

$M_{\max }$ is obtained from

$$
\left.\frac{\partial M}{\partial \sigma_{c}}\right|_{\sigma_{c}=\sigma_{\text {crit }}}=0
$$

Given $n$ we obtain for all $K_{0}$ the following results cribbed fro,n Bludman (1979):

Table 3 - Relativistic Polytropes

\begin{tabular}{|c|l|l|l|}
\hline$n$ & $\Gamma$ & $\sigma_{\text {crit }}$ & $\widetilde{M}$ \\
\hline 0.5 & 3.0 & 1.24 & 0.30 \\
0.6 & 2.7 & 0.97 & 0.28 \\
0.7 & 2.4 & 0.75 & 0.26 \\
0.8 & 2.25 & 0.61 & 0.25 \\
0.9 & 2.1 & 0.50 & 0.25 \\
1.0 & 2 & 0.42 & 0.25 \\
1.5 & 1.67 & 0.20 & 0.28 \\
\hline
\end{tabular}

It is very useful that $\widetilde{M}$ is almost constant at the stability line. Bludman's fit for the onset of instability is 


$$
\Gamma-4 / 3 \simeq 1.73 \sigma_{\text {crit }}-0.31 \sigma_{\text {crit }}^{2}\left(\sigma_{\text {crit }} \leq 2\right)
$$

or

$$
\Gamma-1.5=1.21 \sigma_{\text {crit }}\left(\sigma_{\text {crit }}>\sim 0.4,<1.25\right) .
$$

Note that there are two kinds of polytropic indices

$$
P \sim \varepsilon^{r} \quad P \sim \rho^{\gamma}
$$

related by

$$
\Gamma=\frac{\gamma}{(1+P / \varepsilon)} \Longrightarrow \gamma<\Gamma
$$

I have also done integration of the structure equations using as the EOS

$$
\begin{aligned}
& P=\frac{K_{0}}{9 \gamma} \rho_{0}\left(\frac{\rho}{\rho_{0}}\right)^{\gamma} \\
& \varepsilon=m \rho+\frac{P}{(\gamma-1)} .
\end{aligned}
$$

(In my 1988 paper, there are some simple additional terms to provide the correct symmetry energy at $\rho_{0}$ ). In doing so I made sure to respect causality with

$$
C_{s}^{2}=\frac{\partial P}{\partial \varepsilon} \leq 1 \Longrightarrow P=\varepsilon+\text { const ; }
$$

but this produces not much effect! The results are quite similar to the relativistic case.

A fit to the numerical results, better than $1 \%$, is

$$
M_{\max }=\frac{0.91}{n}\left(\frac{K_{0}}{220}\right)^{n / 2} M_{\odot}
$$

A fit to the maximum central density is

$$
U_{c}=\frac{\rho_{c}}{\rho_{0}}=\left(\gamma-1-0.15 \gamma^{2}\right)\left(\frac{7.1}{M_{\max }}\right)^{2} \text {. }
$$

It is possible to proceed now and talk about what all this has to do with the supernova equation of state, but we'll leave that for other places.

\section{FINAL WORDS}

Well, this is the end of the written lectures, and preparing them has made me think of how many parts of physics and astronomy the subject of supernovae invades. We have not even skimmed the surface of many of the main topics. But 
I hope I have given you some insight into a few of the representative problems. These notes are by no means a complete review, but I hope they give a hook to the interested reader to learn more and help us answer some of the questions.

Preparation also made me think of the summer school itself, the beautiful setting in the Pacific Northwest, and the many hikes, drives, and expeditions whose memories are as immediate as the lectures themselves. An enormous congratulation must be offered to Phil Siemens for the herculean effort he made to organize the summer school, and to make it a total experience. My greatest appreciation must go to the students themslves, who were enthusiastic, hard working, and tried to keep me honest. The staff at Oregon State University also has my strongest thank you. The summer school was made possible by funding from the National Science Foundation.

Much of the material presented here belongs to my collaborators, and I thank them, particularly Ed Baron, for graciously permitting the words to flew from me. 


\section{SUPERNOVA REFERENCE LIST (PARTIAL AND EGOCENTRIC)}

$\underline{\text { EOS }}$

Bethe, ' $/ w n$, Cooperstein; Nucl. Phys. A462 (1987) $791\left[\rho>\rho_{0}\right.$, strange matter $]$

Beth. Irown, Cooperstein, Wilson; Nucl. Phys. A403 (1983) $507\left[\rho<\rho_{0}\right]$

Blaizot, J. P.; Phys. Rep. 64 (1980) 171 [ $K_{0}$ from monopole resonance]

Bludman; ApJ 183637 and 649 (1973) [polytropes]

Cooperstein; Nucl. Phys. $\underline{\mathbf{A} 438}$ (1985) 722-739 $\left[\rho<\rho_{0}\right]$

Cooperstein; in Windsurfing the Fermi Sea vol. 2, Ed by I. T. S.Kuo and J. Speth, North Holland, p. 348 (1987) $\left[\rho>\rho_{0}\right.$, strange matter]

Cooperstein; Phys. Rev. $\underline{\mathrm{C} 37}$ (1988) 786 [neutron stars]

Cooperstein and Wambach; Nucl. Phys. A420 (1984) 591 [electron capture, eos!

Kolehmainen, K., Prakash, M., Lattimer, J., and Treiner, J.;

Nucl. Phys. A439 (1985) 535 [x-dependence of EOS]

Ravenhall, Pethick and Wilson, Phys. Rev. Lett. 150 (1983) 2006

$\left[\rho<\rho_{0}\right.$, geometries $]$

Williams and Koonin, Nucl. Phys. A435 (1985) $844\left[\rho<\rho_{0}\right.$, geometries]

\section{Supernova Theory}

Baron, Bethe, Brown, Cooperstein, Kahana; Phys. Rev. Lett. $\underline{59}$ (1987) 736

Baron, Cooperstein, Kahana; Phys. Rev. Lett. $\underline{55}$ (1985) 126

Baron, Cooperstein, Kahana; Nucl. Phys. A440 (1985) 744

Bethe, Brown, Applegate, Lattimer; Nucl. Phys. A324 (1979) 487

Cooperstein, Bethe, Brown; Nucl. Phys. A429 (1984) 527

\section{Neutrinos}

Cooperstein, Phys. Rep. 163 (1988)

Cooperstein, Van den Horn, Baron; ApJ $\underline{309}$ (1986) 653 [two-fluid model]

Cooperstein, Van den Horn, Baron; ApJ 321 (1987) L129 [pair annihilation]

Van den Horn and Cooperstein; ApJ 300 (1986) 142 [diffusion]

Lattimer and Cooperstein; Phys. Rev. Lett. 61 (1988) 23-26 [magnetic moments]

Self-similar Collapse

Yahil, A.; ApJ. 265 (1983) $1047[\gamma \leq 4 / 3 \mid$

Goldreich, P. and Weber; ApJ. 238 (1980) $991[\gamma=4 / 3]$

\section{DISCLAIMER}

This report was prepared as an account of work sponsored by an agency of the United States Government. Neither the United States Government nor any agency thereof, nor any of their employees, makes any warranty, express or implied, or assumes any legal liability or responsibility for the accuracy, completeness, or usefulness of any information, apparatus, product, or process disclosed, or represents that its use would not infringe privately owned rights. Reference herein to any specific commercial product, process, or service by trade name, trademark, manufacturer, or otherwise does not necessarily constitute or imply its endorsement, recommendation, or favoring by the United States Government or any agency thereof. The views and opinions of authors expressed herein do not necessarily state or reffect those of the United States Government or any agency thereof. 